Review

\title{
Systematic Review of Exposure to Bisphenol A Alternatives and Its Effects on Reproduction and Thyroid Endocrine System in Zebrafish
}

\author{
Jiyun Lee ${ }^{1,2}$, Kyong Whan Moon ${ }^{1,2}$ (D) and Kyunghee $\mathrm{Ji}^{3, *(\mathbb{D})}$ \\ 1 Department of Health and Safety Convergence Science, Graduate School at Korea University, Seoul 02841, \\ Korea; jiyun9607@gmail.com (J.L.); kwmoon@korea.ac.kr (K.-W.M.) \\ 2 BK21 FOUR R\&E Center for Learning Health System, Graduate School at Korea University, Seoul 02841, \\ Korea \\ 3 Department of Occupational and Environmental Health, Yongin University, Yongin 17092, Korea \\ * Correspondence: kyungheeji@yongin.ac.kr; Tel.: +82-31-8020-2747
}

Citation: Lee, J.; Moon, K.W.; Ji, K. Systematic Review of Exposure to Bisphenol A Alternatives and Its Effects on Reproduction and Thyroid Endocrine System in Zebrafish. Appl. Sci. 2021, 11, 1837. https://doi.org/ 10.3390/app11041837

Academic Editor: Miguel Oliveira

Received: 13 January 2021

Accepted: 13 February 2021

Published: 19 February 2021

Publisher's Note: MDPI stays neutra with regard to jurisdictional claims in published maps and institutional affiliations.

Copyright: (c) 2021 by the authors. Licensee MDPI, Basel, Switzerland. This article is an open access article distributed under the terms and conditions of the Creative Commons Attribution (CC BY) license (https:/ / creativecommons.org/licenses/by/ $4.0 /)$.

\begin{abstract}
Bisphenol A (BPA), which is widely used for manufacturing polycarbonate plastics and epoxy resins, has been banned from use in plastic baby bottles because of concerns regarding endocrine disruption. Substances with similar chemical structures have been used as BPA alternatives; however, limited information is available on their toxic effects. In the present study, we reviewed the endocrine disrupting potential in the gonad and thyroid endocrine system in zebrafish after exposure to BPA and its alternatives (i.e., bisphenol AF, bisphenol C, bisphenol F, bisphenol S, bisphenol SIP, and bisphenol Z). Most BPA alternatives disturbed the endocrine system by altering the levels of genes and hormones involved in reproduction, development, and growth in zebrafish. Changes in gene expression related to steroidogenesis and sex hormone production were more prevalent in males than in females. Vitellogenin, an egg yolk precursor produced in females, was also detected in males, confirming that it could induce estrogenicity. Exposure to bisphenols in the parental generation induced a decrease in the hatchability associated with offspring generation. In zebrafish exposed to bisphenols, significant decreases in thyroxine concentrations and increases in thyroidstimulating hormone concentrations were commonly observed. Alternative compounds used to replace a chemical of concern are believed to be less toxic than the original compound; however, several BPA alternatives appear to have similar or greater effects on the endocrine system in zebrafish. Since endocrine systems interact with each other, further studies are needed to assess the primary target of BPA alternatives among the endocrine axes.
\end{abstract}

Keywords: bisphenol A alternatives; endocrine disruption; reproduction; thyroid; zebrafish

\section{Introduction}

The endocrine system comprises endocrine glands, hormones, and target cells involved in reproduction, development, growth, metabolism, and stress response, thereby maintaining the living organism's homeostasis [1]. Hormones produced by the endocrine glands throughout the blood flow circulate in the whole organism and bind to receptors on target cells thus transmitting chemical signals or inducing hormone action. The endocrine system is present in all vertebrates ranging from fish to mammals and secretes similar hormones [2]. Synthetic or natural chemicals that have an abnormal effect on normal endocrine system function are called endocrine disruptors. The exact definition of endocrine disruptors is different for various organizations, such as the United States Environmental Protection Agency [3] or the Organization for Economic Co-operation and Development [4]. Therefore, a globally established list of endocrine disruptors is non-existent, and chemical substances with potential risks are estimated through prior research [5]. Unlike biological hormones, these endocrine disruptors are stable and not easily degraded; therefore, they 
remain in the environment or inside a living organism [6]. Additionally, due to their strong lipid affinity, endocrine disruptors can accumulate in fats and tissues of organisms along the food chain [7]. Chemicals that are estimated to disturb the endocrine system are very diverse, such as plastic plasticizers [8], pesticides [9], heavy metals [10], and persistent organic compounds [11]. Bisphenol A (BPA) is suspected to be a representative endocrine disruptor [12].

BPA is a compound synthesized from phenol and acetone in 1891 [13] and has been used since the 1950s to produce resilient and transparent polycarbonate plastics and epoxy resins [14]. This compound has been used for various purposes, including electronic equipment, medical devices, reusable bottles, food storage containers, canned food, and thermal paper receipts [13,15-17]. The worldwide demand for BPA increases by $5 \%$ annually and is classified as a high production volume chemical in the United States [18]. As the use of BPA increases, it is frequently detected in human samples [19-21], as well as in environmental media $[22,23]$.

As BPA causes estrogenic activity $[24,25]$ and is detected at a high frequency in human, environmental, and product samples, concerns regarding its use have increased. According to the precautionary principle, Canada, the European Union, and the United States have banned the use of BPA in baby bottles [26]. Substances similar in structure to BPA are now used as an alternative [27]. As shown in Table 1, bisphenol analogs have two phenolic rings, and the types and positions of functional groups are slightly different. Since the key structure of bisphenol analogs is similar to the original compound, they can replace the role of BPA. However, there is a possibility that the toxicity resulting from the structure remains. BPA analogs are used in many consumer products and are frequently detected in urine samples of the general population [28], surface water [23], and indoor dust [22].

Table 1. Structural resemblance of bisphenol A analogs.

\begin{tabular}{|c|c|c|c|c|}
\hline Classification & Bisphenol & Bisphenol A (BPA) & Bisphenol AF (BPAF) & Bisphenol C (BPC) \\
\hline \multicolumn{5}{|l|}{ Structure ${ }^{a}$} \\
\hline CAS number & \multirow{3}{*}{$\begin{array}{l}X, Y \text { : regulating factor } \\
\text { for estrogenic andanti-and } \\
\text { rogenic effects }\end{array}$} & $80-05-7$ & $1478-61-1$ & $14868-03-2$ \\
\hline Molecular formula & & $\mathrm{C}_{15} \mathrm{H}_{16} \mathrm{O}_{2}$ & $\mathrm{C}_{15} \mathrm{H}_{10} \mathrm{~F}_{6} \mathrm{O}_{2}$ & $\mathrm{C}_{14} \mathrm{H}_{10} \mathrm{Cl}_{2} \mathrm{O}_{2}$ \\
\hline Molecular weight (g/mol) & & 228.29 & 336.24 & 281.14 \\
\hline Classification & Bisphenol F (BPF) & Bisphenol S (BPS) & Bisphenol SIP (BPSIP) & Bisphenol Z (BPZ) \\
\hline \multicolumn{5}{|l|}{ Structure $^{\text {a }}$} \\
\hline CAS number & $620-92-8$ & 80-09-1 & $95235-30-6$ & $843-55-0$ \\
\hline Molecular formula & $\mathrm{C}_{13} \mathrm{H}_{12} \mathrm{O}_{2}$ & $\mathrm{C}_{12} \mathrm{H}_{10} \mathrm{O}_{4} \mathrm{~S}_{1}$ & $\mathrm{C}_{15} \mathrm{H}_{16} \mathrm{O}_{4} \mathrm{~S}_{1}$ & $\mathrm{C}_{18} \mathrm{H}_{20} \mathrm{O}_{2}$ \\
\hline Molecular weight $(\mathrm{g} / \mathrm{mol})$ & 200.24 & 250.27 & 292.35 & 268.36 \\
\hline
\end{tabular}

The zebrafish has become a powerful model for testing endocrine disruptors based on their higher fecundity, fast embryonic development, and a conserved neuroendocrine system, which are also observed in humans [2]. This small fish has a hypothalamuspituitary-endocrine gland axis, which connects the central nervous system and the endocrine system. Depending on the prominent glands, the axis can be divided into the hypothalamic-pituitary-gonad (HPG) axis, hypothalamic-pituitary-thyroid (HPT) axis, and hypothalamic-pituitary-adrenal (HPA) axis. All axes cooperate with other neuroendocrine systems to control body physiology [2]. Both the technical and practical advantages have 
made zebrafish an ideal organism for the identification of endocrine-disrupting chemicals [29].

As their production and discharge into the environment are estimated to increase worldwide, the health risk potentials of BPA and its alternatives are of increasing concern. Unlike BPA, whose endocrine disruption has been investigated thoroughly, limited information is available regarding the toxicity of BPA alternatives. This review focuses on the endocrine disruption of BPA alternatives in zebrafish that have been reported in previous studies and presents the current status of related knowledge to identify gaps for future research. The first step involves a systematic review to define the protocol through a population-based comparator-outcome (PECO) statement (Table 2). Our review included the literature in which zebrafish were exposed to BPA and its alternatives, with control groups or vehicle-treated groups included for comparison. The effects on the reproduction (fertility, fecundity, vitellogenesis, sex hormones, and genes related to the HPG axis) and development (hatchability, time-to-hatch, spontaneous movement, body length, thyroid hormones, and genes related to hypothalamic-pituitary-thyroid (HPT) axis) were considered as the potential adverse outcomes. The PECO statement serves as a guide for the entire review process, including the literature search strategy, criteria for the inclusion/exclusion of studies, type of data extracted from studies, and strategy for reporting results [25]. 
Table 2. Elements of a population-based comparator-outcome (PECO) statement in the present study.

\begin{tabular}{|c|c|c|c|}
\hline Element & Explanation & Inclusion Criteria & Exclusion Criteria \\
\hline (P) Population & What are the characteristics of the receptors? & - $\quad$ Experimental zebrafish studies & - $\quad$ All humans and rodents studies \\
\hline (E) Exposures & What are the types of chemicals and the timing of exposure? & $\begin{array}{l}\text { - Exposure to BPA, BPAF, BPC, BPF, BPS, BPSIP, and BPZ } \\
\text { in adult stages or early life stages }\end{array}$ & - $\quad$ Exposure to other chemicals \\
\hline (C) Comparator & Which exposure groups will be compared to each other? & $\begin{array}{l}\text { - } \quad \text { Exposed groups versus vehicle-treated or } \\
\text { - } \quad \text { negative controls }\end{array}$ & - No controls in experimental studies \\
\hline (O) Outcome & Which outcomes will be included or covered? & $\begin{array}{l}\text { - Fertility, fecundity, sex ratio, gonad weight, sex } \\
\text { hormones, and genes related to the } \\
\text { hypothalamic-pituitary-gonad (HPG) axis, } \\
\text { vitellogenesis } \\
\text { - Length, weight, thyroid hormones, and genes related to } \\
\text { the hypothalamic-pituitary-thyroid (HPT) axis }\end{array}$ & - All other endocrine disruption effects \\
\hline $\begin{array}{l}\text { Publication } \\
\text { parameters }\end{array}$ & - & $\begin{array}{ll}\text { - } & \text { Peer-reviewed } \\
\text { - } & \text { Original data } \\
& \text { Available in English }\end{array}$ & $\begin{array}{ll}\text { - } & \text { Non-peer reviewed } \\
\text { - } & \text { Not an original data } \\
\text { - } & \text { (e.g., reviews, editorials) } \\
\text { Unavailable in English }\end{array}$ \\
\hline
\end{tabular}




\section{Methods}

\subsection{Search and Selection of Studies for Inclusion}

Relevant studies were selected using two screening phases. The first selection was based on title and abstract screening, and the second selection was based on full-text screening. Studies were selected for full-text screening when they met the inclusion criteria. Articles published before April 1, 2020, were identified in PubMed. A comprehensive search strategy was developed and included the search components "bisphenol A alternatives", "zebrafish", "HPG axis", and "HPT axis". The reference lists of the included articles and relevant reviews were screened manually for potentially relevant new articles. In case of doubt, articles were also analyzed based on their full text. Studies were included in this systematic review when they met all of the following criteria (Table 2): (a) an original full paper that presented unique data; (b) a study where exposure to BPA and its alternatives was elucidated; and (c) an article related to HPG or HPT axes. Studies were excluded if they met one of the following criteria: (a) unoriginal paper, (b) studies involving the exposure to a chemical other than the BPA alternatives, (c) no outcome of interest, and (d) not a zebrafish study. Moreover, selection was restricted to English-language articles.

\subsection{Reliability Assessment of Individual Studies}

The quality of evidence of the systematic review outcomes was rated using the criteria for reporting and evaluating ecotoxicity data (CRED) [24]. A total of 20 criteria were divided into 6 categories for evaluation, including general information, test design, test substance, test organism, exposure conditions, and statistical method. Data above the standard score were judged as high-quality toxicity data. Five points were assigned for each criterion, and the reliability level of the toxicity data was determined based on the satisfaction of 12 essential items and the total score. The CRED evaluation method uses 4 reliability categories, similar to the Klimisch scores [26]: reliable without restrictions (R1), reliable with restrictions (R2), not reliable (R3), and not assignable (R4). R1 grade is given when all 12 essential items are satisfied, and the total score is 80 points or more. The R2 grade is a case where the total score is $60-80$ points, and one of the 12 required items is not satisfied. We selected the data corresponding to R1 and R2 grades.

\section{Results and Discussion}

\subsection{Study Selection and Characteristics}

Study selection is summarized in a flow chart (Figure 1). Literature searches in PubMed identified 40,262 studies related to zebrafish and 24,133 studies related to bisphenols. After removing duplicate records, 241 articles were identified as relevant. After title and abstract screening, 58 articles were excluded based on the following criteria: (a) unavailability in English, (b) unoriginal data, and (c) irrelevant research. Of these 183 publications, 49 were included in this review based on full text screening. 


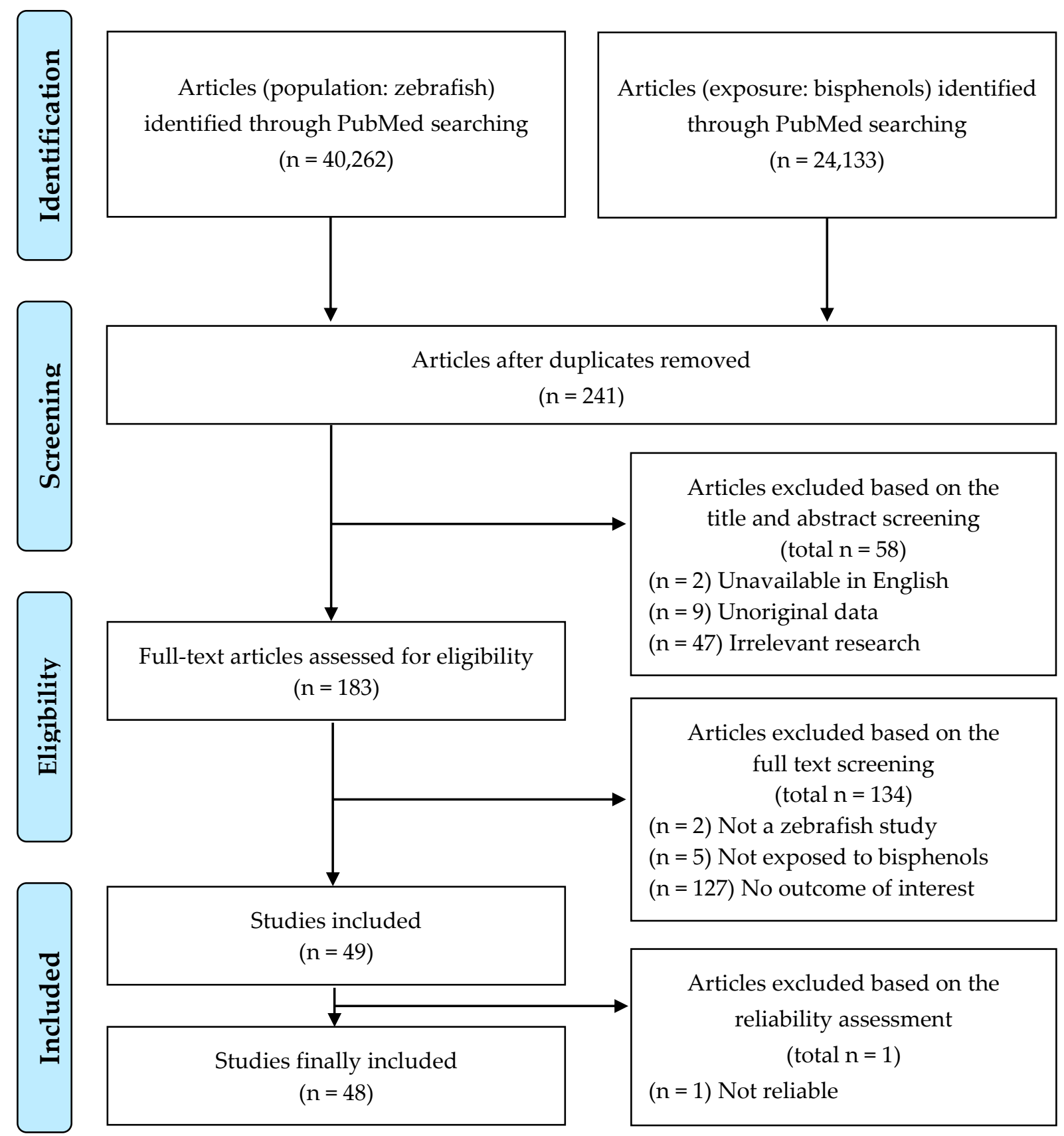

Figure 1. Flow chart of study selection process.

Among 49 individual studies, 9 cases were R1 grade, 39 cases were R2 grade, and 1 case was R3 grade. Therefore, 48 high-quality studies (38 studies related to the HPG axis and 10 studies related to the HPT axis) are summarized in the following section.

\subsection{Effects of BPA and Its Alternatives on HPG Axis}

It has been reported that functions of the reproductive system are susceptible to disruption by endocrine-disrupting chemicals. Thirty-eight studies showed that BPA [27, 28,30-56], BPAF [42,45,51,57,58], BPC [45], BPF [42,46-57,59-61], BPS [42,46-48,62-64], and BPSIP [65] disturbed fecundity, fertility, relative organ weight, vitellogenin protein production, sex hormone production, and mRNA expression related to the HPG axis (Table 3). 
Table 3. Summary of studies about hypothalamic-pituitary-gonad (HPG) axis in zebrafish exposed to bisphenol A and its alternatives.

\begin{tabular}{|c|c|c|c|c|c|c|c|c|c|c|c|}
\hline \multirow[b]{2}{*}{ Chemical } & \multirow[b]{2}{*}{$\begin{array}{l}\text { Stage } \\
\text { (Type) }\end{array}$} & \multirow[b]{2}{*}{$\begin{array}{c}\text { Exposure } \\
\text { Concentration }(\mu \mathrm{g} / \mathrm{L})\end{array}$} & \multirow[b]{2}{*}{$\begin{array}{l}\text { Exposure } \\
\text { Duration }\end{array}$} & \multirow{2}{*}{$\begin{array}{c}\text { No Observed } \\
\text { Adverse } \\
\text { Effect Level } \\
(\mu \mathrm{g} / \mathrm{L})\end{array}$} & \multirow{2}{*}{$\begin{array}{c}\text { Lowest } \\
\text { Observed } \\
\text { Adverse } \\
\text { Effect Level } \\
(\mu \mathrm{g} / \mathrm{L})\end{array}$} & \multicolumn{5}{|c|}{ Toxicity Effect } & \multirow[b]{2}{*}{ Ref } \\
\hline & & & & & & Gene & Hormone & Protein & Organ & Organism & \\
\hline \multirow{3}{*}{ BPA } & \multirow{3}{*}{ Embryo } & \multirow{3}{*}{$0,0.5,5,50,500$} & \multirow{3}{*}{$96 \mathrm{~h}$} & - & 0.5 & $\uparrow e s r 1$, esr $2 a$, vtg 1 & - & - & - & - & \multirow{3}{*}{27} \\
\hline & & & & 0.5 & 5 & $\uparrow c y p 19 a 1 b$ & - & - & - & - & \\
\hline & & & & 50 & 500 & $\uparrow a r$ & - & - & - & - & \\
\hline BPA & Adult & $0,1,10,100,1000$ & 14 days & - & 1 & $\downarrow$ cyp19a (o) & - & $\uparrow$ VTG (o) & - & - & {$[28$} \\
\hline BPA & Adult & $0,100,300,600,800,1000$ & $96 \mathrm{~h}$ & $>1000$ & - & - & - & VTG $\left(0^{\pi}, \uparrow\right)$ & - & - & {$[30$} \\
\hline \multirow{3}{*}{ BPA } & \multirow{3}{*}{ Adult } & \multirow{3}{*}{$0,2,20,200$} & \multirow{3}{*}{21 days } & - & 2 & - & - & 个VTG (\%) & - & - & \multirow{3}{*}{36} \\
\hline & & & & 2 & 20 & - & - & $\uparrow$ VTG $\left(0^{\top}\right)$ & - & - & \\
\hline & & & & 20 & 200 & - & $\begin{array}{c}\downarrow \mathrm{FSH}, \mathrm{LH}, \mathrm{T}, \\
\mathrm{E} 2\left(0^{2}, \stackrel{\uparrow}{ }\right)\end{array}$ & - & - & - & \\
\hline \multirow{3}{*}{ BPA } & \multirow{3}{*}{ Embryo } & \multirow{3}{*}{$\begin{array}{c}0,0.02,0.2,2,22,228,2282 \\
6848 \\
(=0,0.0001,0.001,0.01,0.1,1 \\
10,30 \mu \mathrm{M})\end{array}$} & \multirow{3}{*}{5 days } & 0.2 & 2 & $\downarrow e s r 2$ & - & - & - & - & \multirow{3}{*}{44} \\
\hline & & & & 22 & 228 & Lar & - & - & - & - & \\
\hline & & & & 228 & 2282 & $\downarrow$ tesr1 & - & - & - & - & \\
\hline BPA & Adult & 0,5 & 21 days & - & 5 & $\uparrow$ otg, erd, cyp19a $\left(\sigma^{\top}\right)$ & $\begin{array}{l}\uparrow \mathrm{E} 2\left(\sigma^{\top}\right), \\
\downarrow \mathrm{E} 2(+)\end{array}$ & - & $\downarrow \operatorname{GSI}\left(0^{\top}\right)$ & $\downarrow$ Fecundity & {$[37$} \\
\hline BPA & Adult & $0,10,200,400$ & 180 days & - & 10 & - & - & $\uparrow$ VTG $\left(0^{\top}\right)$ & - & - & {$[39$} \\
\hline \multirow{3}{*}{ BPA } & \multirow{3}{*}{ Embryo } & & & 1 & 10 & $\uparrow g n r h 3, \operatorname{lh} \beta$, kiss1r & - & - & - & - & \\
\hline & & $0,0.1,1,10,100,1000$ & $120 \mathrm{~h}$ & 10 & 100 & $\uparrow e r \alpha$, kiss1 & - & - & - & - & {$[48$} \\
\hline & & & & 100 & 1000 & $\uparrow f \operatorname{sh} \beta$ & - & - & - & - & \\
\hline BPA & Adult & 0,20 & $4 \min$ & - & 20 & $\uparrow \mathrm{F} 1$ vtg 2 & $\downarrow \mathrm{E} 2\left(\left(^{7}\right), \downarrow \mathrm{T}(\%)\right.$ & $\uparrow \mathrm{F} 1 \mathrm{VTG} 2$ & - & $\downarrow$ Fecundity & {$[32$} \\
\hline BPA & Adult & $0,5,10,20$ & 21 days & 10 & 20 & $\uparrow s t a r$, esr $2 b$, fshr (†) & - & - & - & - & {$[50$} \\
\hline BPA & Adult (F2) & 0,20 & 28 days & - & 20 & $\uparrow s t a r, f s h r($ ( ) & - & - & $\downarrow$ GSI (†) & $\downarrow$ Fertility & {$[49$} \\
\hline BPA & Embryo & 0,100 & $120 \mathrm{~h}$ & - & 100 & $\uparrow e r \alpha$ & - & - & - & - & {$[47$} \\
\hline BPA & Juvenile & 0,100 & 60 days & - & 100 & 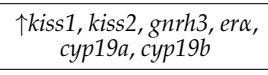 & - & - & - & - & {$[46$} \\
\hline & & $0,10,100,1000$ & 15 davs & 10 & 100 & 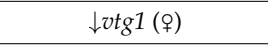 & - & - & - & - & {$[40$} \\
\hline BPA & Adult & $0,10,100,1000$ & 15 days & 100 & 1000 & $\uparrow v \operatorname{tg} 1\left(\sigma^{\pi}\right)$ & - & - & - & - & {$[40$} \\
\hline & & 200 200 & 42 days & - & 100 & $\uparrow$ otg1(laiơ $)$ & - & - & $\downarrow$ GSI $\left(0^{7}\right)$ & - & {$[52$} \\
\hline BPA & Adult & $0,100,2000$ & 42 days & 100 & 2000 & $\uparrow e s r 1$, vtg2 $\left(\sigma^{\top}\right)$ & - & - & - & - & {$[52$} \\
\hline
\end{tabular}


Table 3. Cont.

\begin{tabular}{|c|c|c|c|c|c|c|c|c|c|c|c|}
\hline \multirow[b]{2}{*}{ Chemical } & \multirow[b]{2}{*}{$\begin{array}{l}\text { Stage } \\
\text { (Type) }\end{array}$} & \multirow[b]{2}{*}{$\begin{array}{c}\text { Exposure } \\
\text { Concentration }(\mu \mathrm{g} / \mathrm{L})\end{array}$} & \multirow[b]{2}{*}{$\begin{array}{l}\text { Exposure } \\
\text { Duration }\end{array}$} & \multirow{2}{*}{$\begin{array}{c}\text { No Observed } \\
\text { Adverse } \\
\text { Effect Level } \\
(\mu \mathrm{g} / \mathrm{L})\end{array}$} & \multirow{2}{*}{$\begin{array}{c}\text { Lowest } \\
\text { Observed } \\
\text { Adverse } \\
\text { Effect Level } \\
(\mu \mathrm{g} / \mathrm{L})\end{array}$} & \multicolumn{5}{|c|}{ Toxicity Effect } & \multirow[b]{2}{*}{ Ref } \\
\hline & & & & & & Gene & Hormone & Protein & Organ & Organism & \\
\hline \multirow{3}{*}{ BPA } & \multirow{3}{*}{ Embryo } & \multirow{3}{*}{$0,100,1000,5000$} & \multirow{3}{*}{$96 \mathrm{~h}$} & - & 100 & $\uparrow$ otg1, сур $17 a 1$ & - & - & - & - & \multirow{3}{*}{ [42] } \\
\hline & & & & 100 & 1000 & $\uparrow e s r 1$, esr $2 a$, esr $2 b, h s d 17 b 1$ & - & - & - & - & \\
\hline & & & & 1000 & 5000 & $\uparrow c y p 19 a 1$ & - & - & - & - & \\
\hline BPA & Adult & $0,25,50,100,250,500$ & 15 days & 100 & 250 & $\uparrow v \operatorname{tg} 1\left(\sigma^{\top}\right)$ & - & - & - & - & {$[31$} \\
\hline BPA & Adult & $0,500,1000,1500$ & 21 days & - & 500 & - & - & $\uparrow$ VTG $\left(0^{\top}\right)$ & - & - & {$[51$} \\
\hline \multirow[b]{2}{*}{ BPA } & \multirow[b]{2}{*}{ Adult } & \multirow[b]{2}{*}{$0,500,1000,1500$} & \multirow[b]{2}{*}{21 days } & - & 500 & $\uparrow v t g 1$, esr2b, cyp19a1a $\left(\sigma^{\top}\right)$ & $\uparrow \mathrm{E} 2\left(\sigma^{7}\right)$ & $\uparrow$ VTG $\left(0^{7}\right)$ & - & - & \multirow[b]{2}{*}{56} \\
\hline & & & & 500 & 1000 & 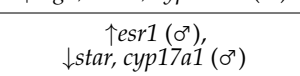 & $\downarrow \mathrm{T}\left(\sigma^{\top}\right)$ & 1 & - & - & \\
\hline \multirow{2}{*}{ BPA } & \multirow{2}{*}{ Embryo } & \multirow{2}{*}{$\begin{array}{c}0,10,100,500,750 \\
1000,2500,5000\end{array}$} & \multirow{2}{*}{7 days } & 500 & 750 & $\uparrow c y p 19 b$ & - & - & - & - & \multirow{2}{*}{ [55] } \\
\hline & & & & 1000 & 2500 & $\uparrow$ vtg & - & - & - & - & \\
\hline BPA & Adult & $0,40,200,1000$ & 21 days & 200 & 1000 & - & - & 个VTG (ơ) & - & - & {$[53$} \\
\hline BPA & Adult & 0,2000 & 35 days & - & 2000 & $\uparrow v t g\left(\sigma^{\pi}\right)$ & - & - & - & - & {$[41$} \\
\hline BPA & Embryo & $\begin{array}{l}0,1141,2282,3424 \\
(=0,5,10,15 \mu \mathrm{M})\end{array}$ & $120 \mathrm{~h}$ & 1141 & 2282 & $\uparrow v t g 1$ & - & - & - & - & {$[43$} \\
\hline BPA & Embryo(GFP) & $\begin{array}{c}0,11,22,114,228,1141,2282 \\
(=0,0.05,0.1,0.5,1,5,10 \\
\mu \mathrm{M})\end{array}$ & 2 days & 1141 & 2282 & $\uparrow$ otg1, cyp19a1b & - & - & - & - & {$[45$} \\
\hline BPA & Juvenile(albino) & $0,2282(=10 \mu \mathrm{M})$ & 20 days & - & 2282 & $\downarrow f \operatorname{sh} \beta$ & - & - & $\begin{array}{l}\downarrow \text { Ovary } \\
\text { growth }\end{array}$ & $\uparrow q / o^{\top}$ ratio & {$[34$} \\
\hline ВРА & Embryo & $\begin{array}{c}0,114,228 \\
570,1141,2282 \\
(=0,0.5,1,2.5,5,10 \mu \mathrm{M})\end{array}$ & 7 days & $>2282$ & - & $\operatorname{vtg} 1$ & - & - & - & - & {$[33$} \\
\hline BPA & Embryo & $0,804,2010,4020,6030$ & $96 \mathrm{~h}$ & 4020 & 6030 & $\uparrow$ vtg1 & - & - & - & - & {$[35$} \\
\hline BPA & Adult & $\begin{array}{c}0,0.1,2,20,200,400,1000 \\
2000\end{array}$ & 11 days & 1000 & 2000 & $\uparrow v t g 1\left(\odot^{\top}\right)$ & - & - & - & - & {$[38$} \\
\hline \multirow{3}{*}{ BPAF } & \multirow{3}{*}{ Embryo } & \multirow{3}{*}{$0,20,200,1000$} & \multirow{3}{*}{$96 \mathrm{~h}$} & - & 20 & $\uparrow v t g 1, e s r 2 b$ & - & - & - & - & \multirow{3}{*}{42} \\
\hline & & & & 20 & 200 & $\begin{array}{c}\text { Tesr1, esr2a, cyp19a1, } \\
\text { hsd17b1 }\end{array}$ & - & - & - & - & \\
\hline & & & & 200 & 1000 & $\uparrow c y p 17 a 1$ & - & - & - & - & \\
\hline \multirow{5}{*}{ BPAF } & \multirow{5}{*}{ Adult } & & & - & 5 & 10 & $\uparrow \mathrm{E} 2\left(\sigma^{7}\right)$ & 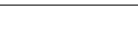 & 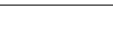 & 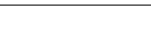 & \\
\hline & & & & 5 & 25 & Totg $1\left(0^{\prime \prime}\right)$ & $\downarrow \mathrm{T}\left(0^{\top}\right)$ & - & - & - & \\
\hline & & $0,5,25,125$ & 120 days & & & $\begin{array}{l}\uparrow g n r h 2, \text { fshl } \beta, \operatorname{lh} \beta, \text { fshr, star, } \\
\text { cyp17, cyp } 19 a, \text { cypp19b }\left(\sigma^{\prime}\right)\end{array}$ & & & & & {$[57$} \\
\hline & & & & 25 & 125 & $\uparrow$ fshr (q) & $\uparrow \mathrm{E} 2$ (o) & - & - & $\downarrow$ F1 fertility & \\
\hline & & & & & & $\downarrow$ star (\%) & & & & & \\
\hline
\end{tabular}


Table 3. Cont.

\begin{tabular}{|c|c|c|c|c|c|c|c|c|c|c|c|}
\hline \multirow[b]{2}{*}{ Chemical } & \multirow[b]{2}{*}{$\begin{array}{l}\text { Stage } \\
\text { (Type) }\end{array}$} & \multirow[b]{2}{*}{$\begin{array}{c}\text { Exposure } \\
\text { Concentration }(\mu \mathrm{g} / \mathrm{L})\end{array}$} & \multirow[b]{2}{*}{$\begin{array}{l}\text { Exposure } \\
\text { Duration }\end{array}$} & \multirow{2}{*}{$\begin{array}{l}\text { No Observed } \\
\text { Adverse } \\
\text { Effect Level } \\
(\mu \mathrm{g} / \mathrm{L})\end{array}$} & \multirow{2}{*}{$\begin{array}{c}\text { Lowest } \\
\text { Observed } \\
\text { Adverse } \\
\text { Effect Level } \\
(\mu \mathrm{g} / \mathrm{L})\end{array}$} & \multicolumn{5}{|c|}{ Toxicity Effect } & \multirow[b]{2}{*}{ Ref } \\
\hline & & & & & & Gene & Hormone & Protein & Organ & Organism & \\
\hline \multirow{2}{*}{ BPAF } & \multirow{2}{*}{ Embryo(GFP) } & \multirow{2}{*}{$\begin{array}{c}0,16,33,168,336,1681 \\
(=0,0.05,0.1,0.5,1,5 \mu \mathrm{M})\end{array}$} & \multirow{2}{*}{2 days } & 33 & 168 & $\uparrow c y p 19 a 1 b$ & - & - & - & - & \multirow{2}{*}{ [45] } \\
\hline & & & & 168 & 336 & $\uparrow$ vtg1 & - & - & - & - & \\
\hline BPAF & Adult & $0,500,1000,1500$ & 21 days & - & 500 & - & - & $\uparrow$ VTG $\left(0^{\pi}\right)$ & - & - & [51] \\
\hline BPAF & Adult & $0,50,250,1000$ & 28 days & 250 & 1000 & $\uparrow v \operatorname{tg}\left(0^{\pi}\right)$ & $\uparrow \mathrm{E} 2\left(0^{\top}\right)$ & - & - & - & [58] \\
\hline BPC & Embryo(GFP) & $\begin{array}{c}0,14,28,140,281,1405 \\
(=0,0.05,0.1,0.5,1,5 \mu \mathrm{M})\end{array}$ & 2 days & - & 14 & †⿰tg1 & - & - & - & - & [45] \\
\hline \multirow{5}{*}{ BPF } & \multirow{5}{*}{ Juvenile } & \multirow{5}{*}{$0,0.1,1,10,100,1000$} & \multirow{5}{*}{60 days } & - & 0.1 & - & - & $\uparrow$ VTG & - & - & \multirow{5}{*}{ [46] } \\
\hline & & & & 1 & 10 & 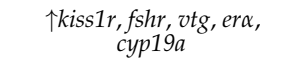 & $\begin{array}{l}\text { 个LH, FSH, } \\
\text { GnRH }\end{array}$ & - & - & - & \\
\hline & & & & 10 & 100 & $\uparrow k i s s 1, l h r$ ( (), er $\beta$, cyp19b & - & - & - & - & \\
\hline & & & & 100 & 1000 & $\uparrow g n r h 3, \operatorname{lhr}\left(0^{7}\right)$ & - & - & - & - & \\
\hline & & & & $>1000$ & - & kiss $2, k i s s 2 r, s v 2 c$ & - & - & GSI (o) & - & \\
\hline \multirow{8}{*}{ BPF } & \multirow{8}{*}{ Adult } & \multirow{8}{*}{$0,1,10,100,1000$} & \multirow{8}{*}{21 days } & - & 1 & $\uparrow f s h \beta\left(\sigma^{\top}\right)$ & - & - & - & - & \\
\hline & & & & 1 & 10 & $\uparrow \ln \beta, \operatorname{gnrh} 3, \operatorname{vtg}\left(\sigma^{\top}\right)$ & $\downarrow T\left(0^{7}\right)$ & - & - & - & \\
\hline & & & & \multirow{3}{*}{10} & \multirow{3}{*}{100} & $\begin{array}{l}\text { Tgnrh2, gnrhr1, gnrhr2, } \\
\text { cyp19a, fshr, lhr }\left(\left(^{\top}\right)\right)\end{array}$ & & & & & \\
\hline & & & & & & $\uparrow$ fshr $($ ( ) & $\uparrow \mathrm{E} 2\left(0^{\top}\right)$ & - & - & - & [61] \\
\hline & & & & & & $\downarrow_{f s h} \beta, 17 \beta h s d$, star (o) & & & & & \\
\hline & & & & & & $\uparrow \operatorname{cyp} 11 a\left(0^{\pi}\right.$, ᄋ) & & & & & \\
\hline & & & & 100 & 1000 & $\downarrow 17 \beta h s d$, cyp 17, star $\left(0^{\top}\right)$ & 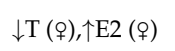 & - & $\downarrow \mathrm{GSI}\left(0^{\pi}\right.$, ㅇ) & $\begin{array}{l}\downarrow \text { Fecundity, } \\
\text { F1 survival }\end{array}$ & \\
\hline & & & & & & $\downarrow l h r($ ( ) & & & & & \\
\hline & & & & - & 1 & 个cyp19a1a, vtg & - & - & - & - & \\
\hline BPF & Juvenile & $0,1,10,100,1000$ & 60 days & 1 & 10 & $\downarrow a m h$, foxl2 & 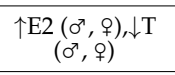 & - & - & - & [60] \\
\hline & & & & 10 & 100 & $\uparrow d m r t 1$ & - & - & - & $\uparrow$ Intersex & \\
\hline & & & & 100 & 1000 & $\downarrow f f 1 d$ & - & - & - & - & \\
\hline BPF & Adult(GFP) & $\begin{array}{c}0,2.28,22.8,228,2282(=0 \\
0.01,0.1,1,10 \mu \mathrm{M})\end{array}$ & 7 days & 2.28 & 22.8 & - & - & $\uparrow$ VTG $\left(\sigma^{\top}\right)$ & - & - & [59] \\
\hline BPF & Embryo & $0,0.1,1,10,100,1000$ & $120 \mathrm{~h}$ & 10 & 100 & 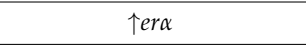 & - & - & - & - & [47] \\
\hline & & & & 200 & 2000 & $\uparrow$ otg1 & - & - & - & - & \\
\hline BPF & Embryo & $0,200,2000,10,000$ & $96 \mathrm{~h}$ & 2000 & 10,000 & $\begin{array}{c}\text { 个esr1, esr2a, esr2b, cyp19a1, } \\
\text { cyp17a1, hsd17b1 }\end{array}$ & - & - & - & - & [42] \\
\hline
\end{tabular}


Table 3. Cont

\begin{tabular}{|c|c|c|c|c|c|c|c|c|c|c|c|}
\hline \multirow[b]{2}{*}{ Chemical } & \multirow[b]{2}{*}{$\begin{array}{l}\text { Stage } \\
\text { (Type) }\end{array}$} & \multirow[b]{2}{*}{$\begin{array}{c}\text { Exposure } \\
\text { Concentration }(\mu \mathrm{g} / \mathrm{L})\end{array}$} & \multirow[b]{2}{*}{$\begin{array}{l}\text { Exposure } \\
\text { Duration }\end{array}$} & \multirow{2}{*}{$\begin{array}{c}\text { No Observed } \\
\text { Adverse } \\
\text { Effect Level } \\
(\mu \mathrm{g} / \mathrm{L})\end{array}$} & \multirow{2}{*}{$\begin{array}{c}\text { Lowest } \\
\text { Observed } \\
\text { Adverse } \\
\text { Effect Level } \\
(\mu \mathrm{g} / \mathrm{L})\end{array}$} & \multicolumn{5}{|c|}{ Toxicity Effect } & \multirow[b]{2}{*}{ Ref } \\
\hline & & & & & & Gene & Hormone & Protein & Organ & Organism & \\
\hline \multirow{3}{*}{ BPS } & \multirow{3}{*}{ Adult } & \multirow{3}{*}{$0,0.5,5,50$} & \multirow{3}{*}{21 days } & - & 0.5 & - & $\uparrow \mathrm{E} 2\left(\sigma^{\top}\right)$ & - & $\downarrow$ GSI (†) & $\downarrow$ Fecundity & \multirow{3}{*}{62} \\
\hline & & & & \multirow[t]{2}{*}{5} & \multirow[t]{2}{*}{50} & 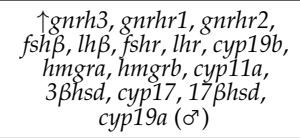 & $\downarrow T\left(0^{\top}\right)$ & - & $\downarrow$ GSI $\left(0^{\top}\right)$ & - & \\
\hline & & & & & & 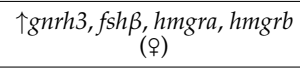 & $\uparrow \mathrm{E} 2$ (\%) & & & & \\
\hline BPS & Adult & $0,1,10,30$ & 120 days & - & 1 & $\uparrow e s r 2 a$, esr $2 b$ (o) & - & - & - & - & [63] \\
\hline \multirow{3}{*}{ BPS } & \multirow{3}{*}{ Adult } & \multirow{3}{*}{$0,0.1,1,10,100$} & \multirow{3}{*}{75 days } & 0.1 & 1 & - & $\uparrow \mathrm{E} 2\left(0^{\top}\right)$ & - & - & - & \multirow{3}{*}[64]{} \\
\hline & & & & 1 & 10 & - & $\downarrow \mathrm{T}\left(0^{\top}\right), \uparrow \mathrm{E} 2(\%)$ & 个VTG (\$) & $\downarrow G S I\left(\sigma^{7}\right)$ & $\downarrow$ Fecundity & \\
\hline & & & & 10 & 100 & - & & 个VTG $\left(0^{\uparrow}\right)$ & $\downarrow \mathrm{GSI}$ (†) & - & \\
\hline BPS & Embryo & 0,100 & $120 \mathrm{~h}$ & 10 & 100 & $\uparrow g n r h 3, k i s s 1$, era & - & - & - & - & [48] \\
\hline BPS & Embryo & $0,0.1,1,10,100,1000$ & $120 \mathrm{~h}$ & 10 & 100 & $\uparrow e r \alpha$ & - & - & - & - & [47] \\
\hline BPS & Juvenile & 0,100 & 60 days & - & 100 & 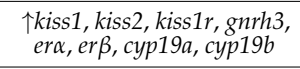 & - & - & - & - & [46] \\
\hline BPS & Embryo & $0,500,5000,25,000$ & $96 \mathrm{~h}$ & $>25,000$ & - & $\begin{array}{c}\text { vtg1, esr1, esr2a, esr } 2 b, \\
\text { cyp19a1, cyp17a1, hsd17b1 }\end{array}$ & - & - & - & - & [42] \\
\hline \multirow{3}{*}{ BPSIP } & \multirow{3}{*}{ Adult } & \multirow{3}{*}{$0,0.5,5,50$} & \multirow{3}{*}{21 days } & \multirow{3}{*}{5} & \multirow{3}{*}{50} & 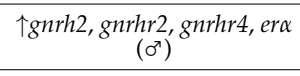 & $\downarrow \mathrm{E} 2\left(\sigma^{\pi}\right)$ & \multirow{3}{*}{-} & \multirow{3}{*}{-} & \multirow{3}{*}{-} & \multirow{3}{*}{65} \\
\hline & & & & & & $\downarrow$ fsh $\beta$, cyp $17,17 \beta h s d\left(\sigma^{\top}\right)$ & & & & & \\
\hline & & & & & & 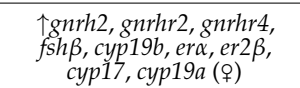 & $\uparrow \mathrm{E} 2, \mathrm{~T}$ () & & & & \\
\hline
\end{tabular}




\subsubsection{Effects on Reproduction}

The reproductive process in fish is regulated by steroidogenesis and coordinated interactions between steroid hormones on the HPG axis [66]. Therefore, chemicals that alter the concentration of sex hormones and the expression of steroidogenesis-related genes could affect the endocrine system's functionality, eventually influencing the reproduction of fish [67]. Exposure to bisphenols in the parental generation induced a significant decrease in fecundity $[32,37,61,62,64]$. Parental exposure to bisphenols is essential because no excretion mechanism exists in the eggs [62]. One explanation for the reproductive effect of parental generation and the developmental effect of offspring generation is the change in hormones and genes in the HPG axis.

\subsubsection{Effects on Relative Gonad Weight}

The gonadosomatic index (GSI), which is the ratio of the gonad weight to body weight, has been used as a biomarker for endocrine disruption. GSI indicates the effects on the development of reproductive organs along with a decrease in fecundity and a change in sex ratio [68]. Male zebrafish were more sensitive to exposure to bisphenols than females. Male zebrafish exposed to BPA, BPF, BPS, and BPSIP significantly decreased GSI at 5-100 $\mu \mathrm{g} / \mathrm{L}$ [37,52], $1000 \mu \mathrm{g} / \mathrm{L}$ [61], 10-50 $\mu \mathrm{g} / \mathrm{L}$ [62,64], and $5 \mu \mathrm{g} / \mathrm{L}$ [65], respectively (Figure 2). Several studies have reported that estrogenic chemicals reduce GSI by altering the number and size of germ cells in zebrafish $[69,70]$. These observations indicate that alternatives such as BPS and BPSIP can affect gonadal development, which is similar to BPA.

LOAEL of gonadosomatic index in male zebrafish

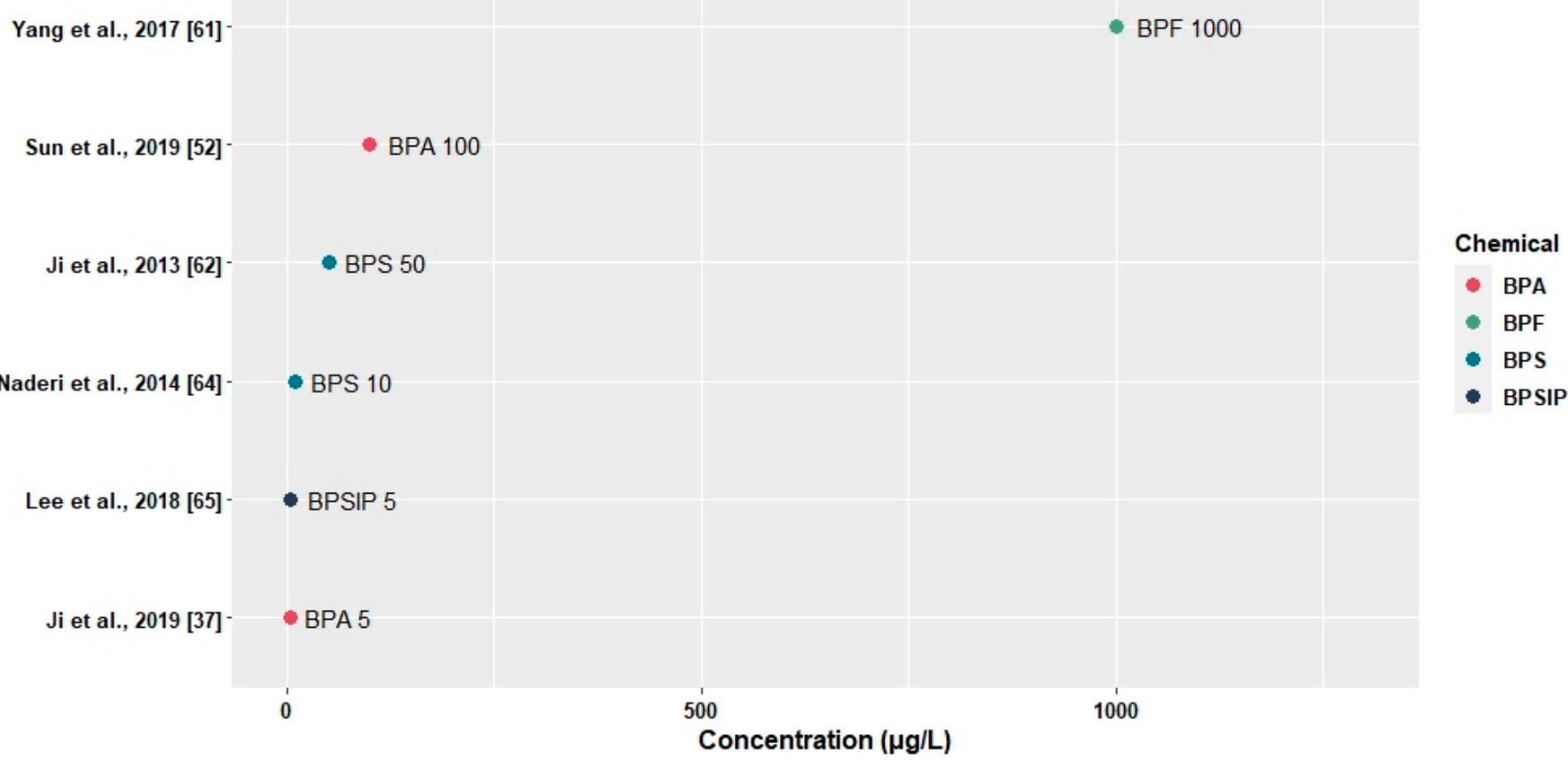

Figure 2. Lowest observed adverse effect level (LOAEL) of gonadosomatic index (GSI) in male zebrafish exposed to BPA, $\mathrm{BPF}, \mathrm{BPS}$, and BPSIP.

\subsubsection{Effects on Vitellogenesis}

Vitellogenin is a yolk precursor expressed in females and is used as a biomarker for endocrine disruptors. Figure 3 shows the studies on vitellogenin protein production and vitellogenin gene transcription in male zebrafish exposed to BPA [31,37,38,40,41,52,56], BPAF [57,58], and BPF [61]. Male zebrafish exposed to BPA, BPAF, BPF, and BPS signifi- 
cantly increased vitellogenin production at 10-1000 $\mu \mathrm{g} / \mathrm{L}$ [36,39,51,53,54,56], $500 \mu \mathrm{g} / \mathrm{L}$ [51], $22.8 \mu \mathrm{g} / \mathrm{L}$ [59], and $100 \mu \mathrm{g} / \mathrm{L}$ [64], respectively. In male zebrafish exposed to BPA, $\mathrm{BPAF}$, and BPF, vitellogenin mRNA expression was significantly increased at 5-2000 $\mu \mathrm{g} / \mathrm{L}[31,37,38,40,41,52,56], 25-1000 \mu \mathrm{g} / \mathrm{L}$ [57,58], and $10 \mu \mathrm{g} / \mathrm{L}$ [61], respectively. These studies demonstrate that it is possible to induce endocrine disruption at a detectable concentration in aquatic environments [71].

(a)

LOAEL of VTG protein production in male zebrafish

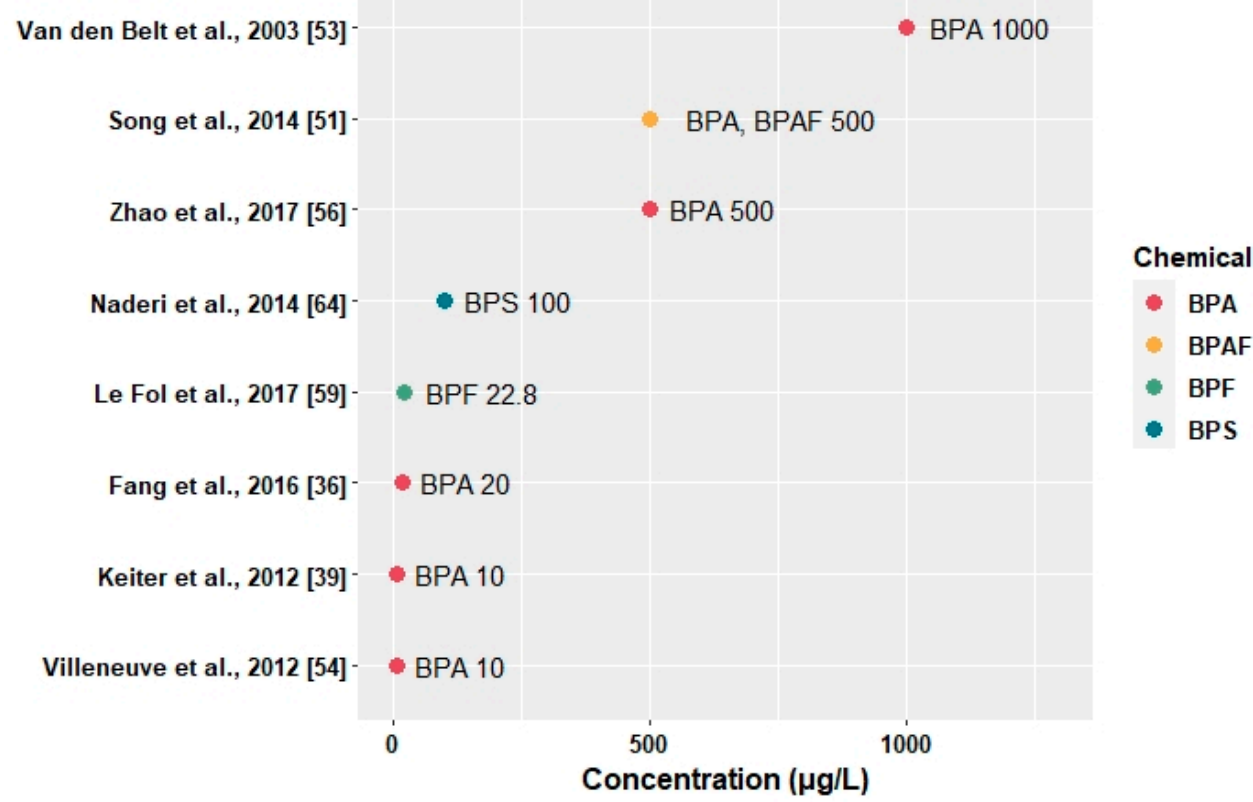

(b)

LOAEL of vtg mRNA expression in male zebrafish

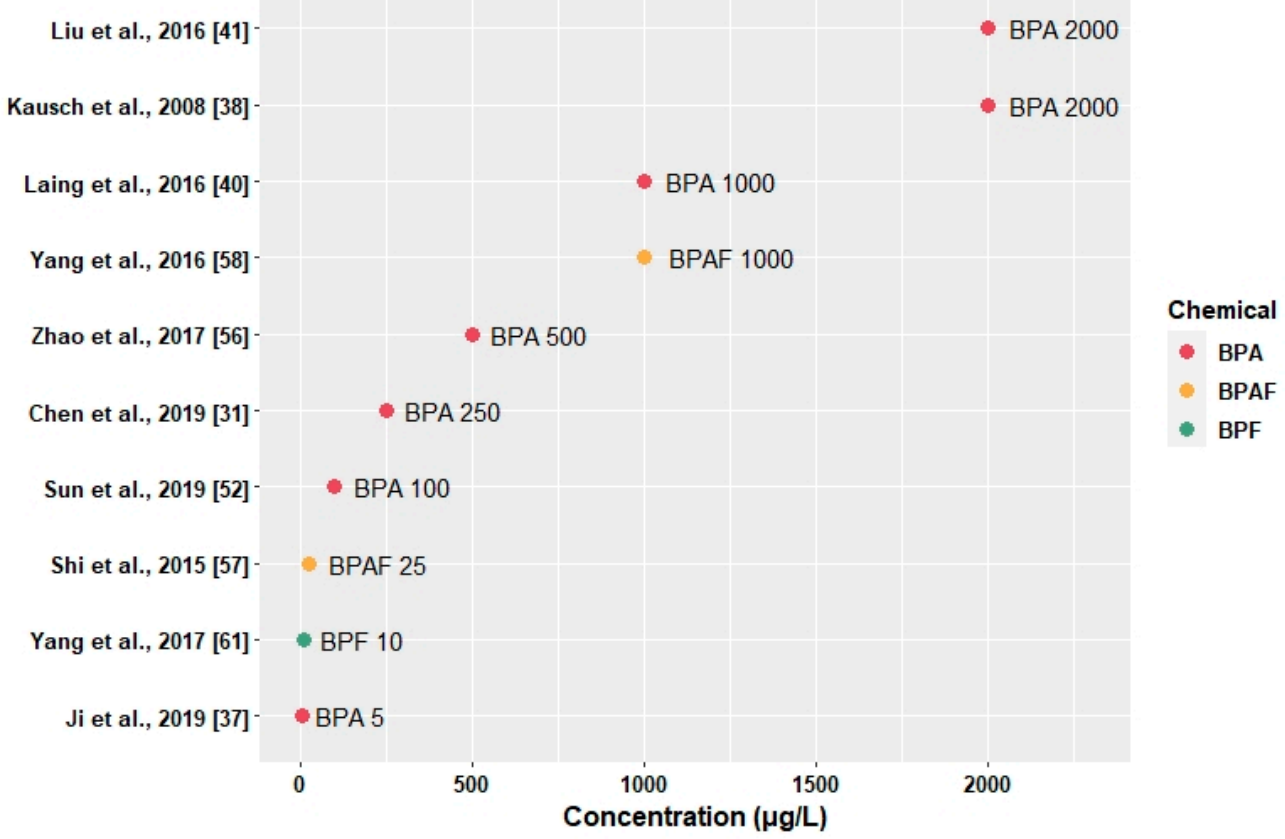

Figure 3. Lowest observed adverse effect level (LOAEL) of vitellogenin protein production (a) and vitellogenin mRNA expression (b) in male zebrafish exposed to BPA, BPAF, BPF, and BPS. 


\subsubsection{Effects on Sex Hormones and Genes Related to HPG Axis}

The concentration and ratio of $17 \beta$-estradiol (E2) and testosterone (T) hormones have been widely used as integrated biomarkers for reproduction. In the studies summarized here, the adverse effects of BPA and its alternatives on hormone levels were sex-dependent, and a significant increase in E2 and decrease in T concentrations were observed in male zebrafish exposed to BPA, BPAF, BPF, BPS, and BPSIP (Figure 4). Interestingly, changes in sex hormones in male zebrafish were more sensitive to the toxicity of its alternatives than BPA. For example, male zebrafish exposed to BPS significantly increased E2 compared to the control group even at $0.5-1 \mu \mathrm{g} / \mathrm{L}[62,64]$. The decrease in T hormone was up to 200 times more sensitive than BPA for most alternatives (e.g., BPAF, BPF, BPS, and BPSIP) [57,60$62,64,65]$. These results suggest that the endocrine disruption of BPA alternatives is no less than that of BPA and may significantly affect sex hormones in males.

(a) LOAEL of E2 hormone regulation in male zebrafish

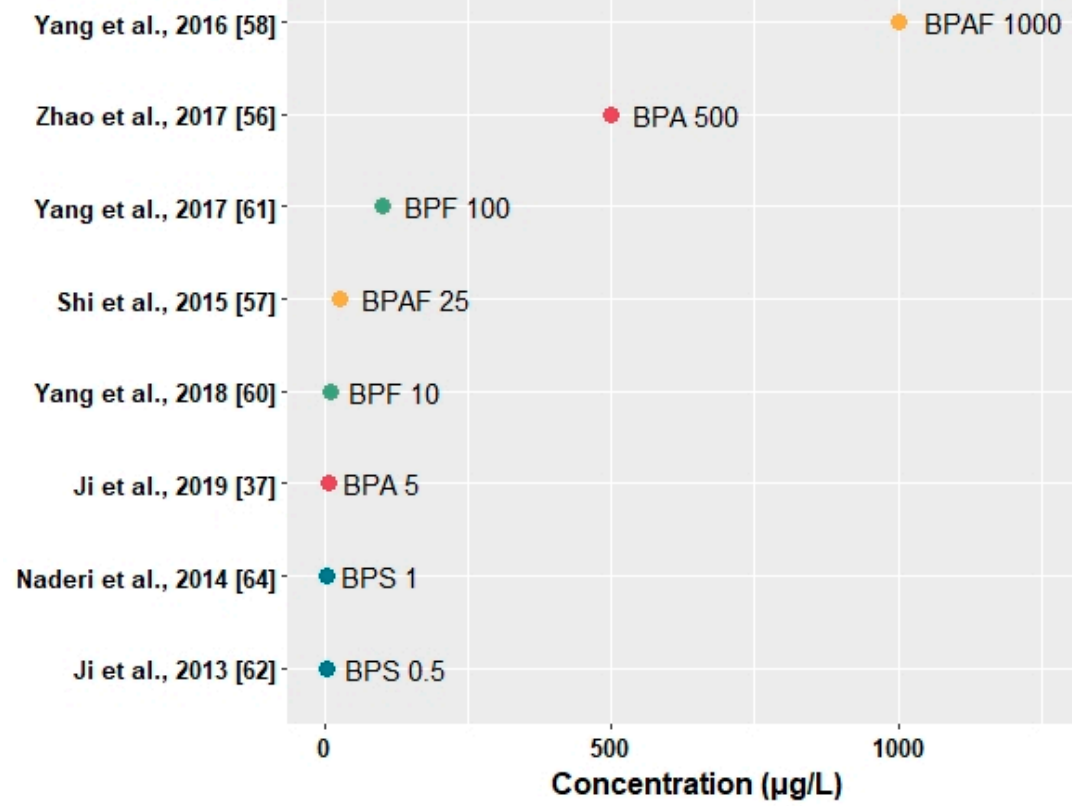

(b)

LOAEL of $T$ hormone regulation in male zebrafish

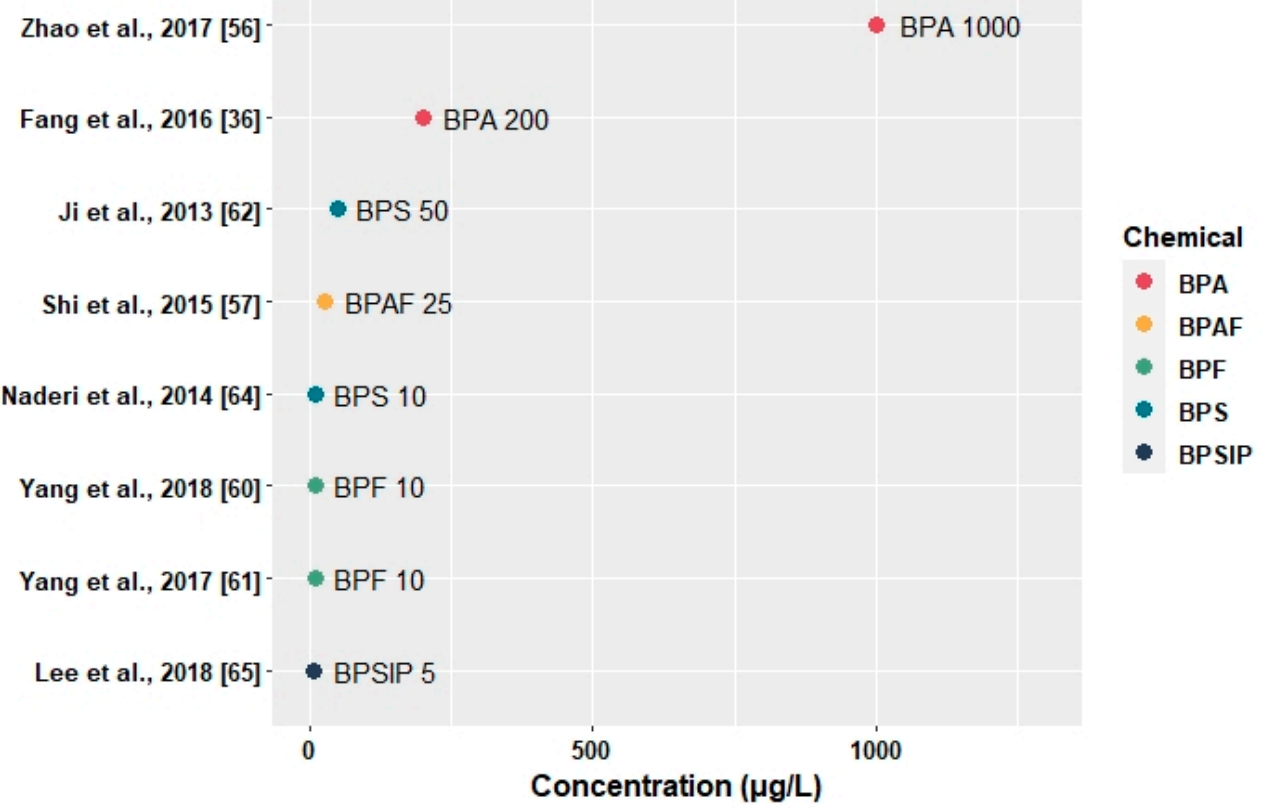

Figure 4. Cont. 
(c) LOAEL of cyp19a mRNA expression in male zebrafish

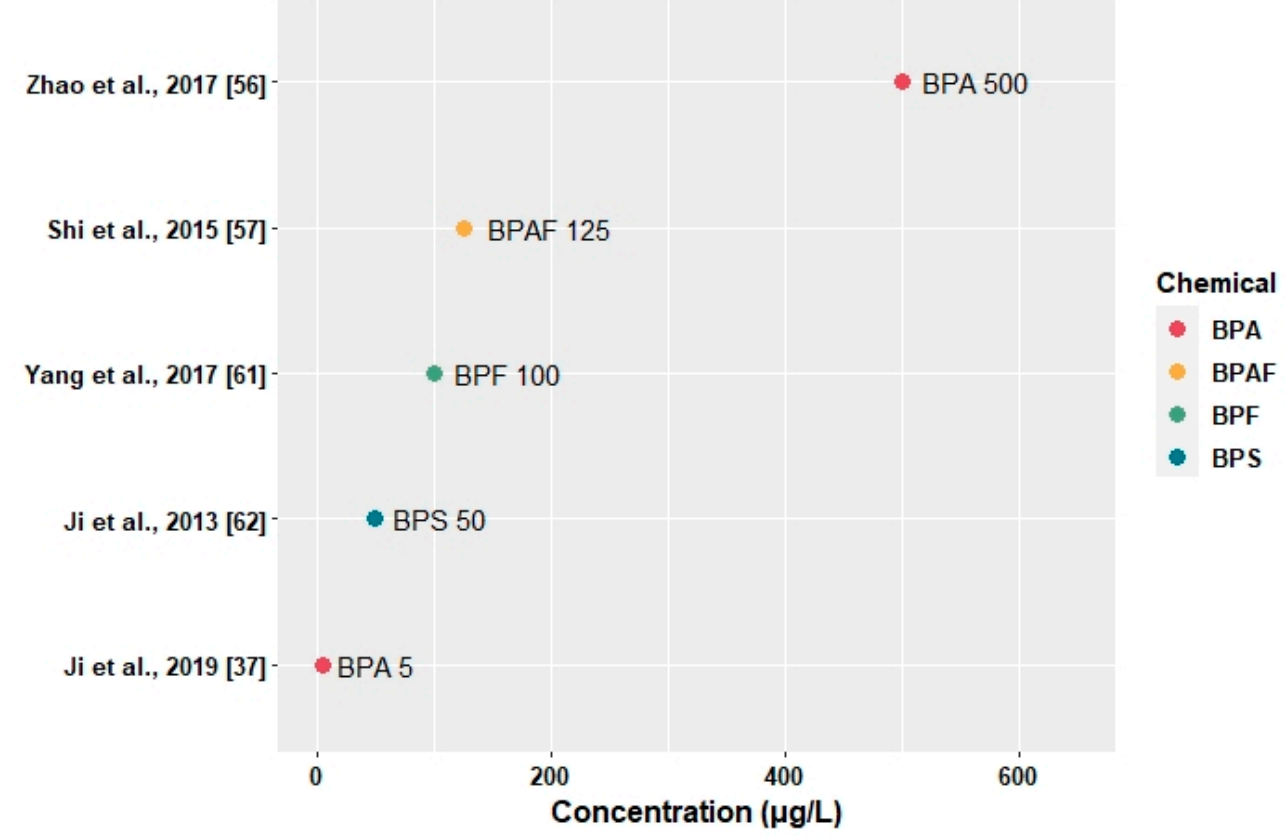

Figure 4. Lowest observed adverse effect level (LOAEL) of 17 $\beta$-estradiol (E2) hormone (a), testosterone (T) hormone (b), and cyp19a mRNA expression (c) in male zebrafish exposed to BPA, BPAF, BPF, BPS, and BPSIP.

Significant increases in aromatase (cyp19) genes are well supported by changes in the two sex hormones. CYP19 enzyme is involved in the final step in converting of $\mathrm{T}$ to E2 [72], and enzyme activities are generally well correlated with their mRNA levels [73]. Cyp19a mRNA expression increased significantly in male zebrafish exposed to BPA [37,56], BPAF [57], BPF [61], and BPS [62] (Figure 4). Interestingly, the adverse effects of BPA alternatives on gene transcription were sex-dependent, with males being more sensitive than females.

In the HPG axis, gonadotropin-releasing hormone $(\mathrm{GnRH})$ in the hypothalamus stimulates luteinizing hormone (LH) and follicle-stimulating hormone (FSH) in teleosts [74]. Gonadotropin hormones in the pituitary regulate the synthesis of sex hormones, E2 and T. In terms of homeostasis, it is meaningful to examine the effects of BPA and its alternatives on the GnRH, LH, and FSH of zebrafish. Zebrafish possess the hormones GnRH2 and GnRH3, as well as four different GnRH receptors [75]. While there are few studies are measuring the GnRH hormone [46], it was frequently confirmed that the expression of gnrh2 or gnrh3 genes in zebrafish exposed to BPA, BPAF, BPF, BPS, and BPSIP was significantly increased (Table 3) $[46,48,57,61,62,65]$. These results suggest that BPA analogs can directly or indirectly (e.g., a negative feedback action in the hypothalamus compensates for the reduced E2 production) affect GnRH.

$\mathrm{LH}$ and FSH, which bind to specific receptors and induce gametogenesis, were also confirmed by measuring hormone or gene expression in zebrafish exposed to BPA and its alternatives. The $\beta$-subunit mRNA encoding LH and FSH were generally upregulated in male zebrafish exposed to BPAF [57], BPF [61], and BPS [62]. Two possibilities can be responsible for the decrease in the $\mathrm{T}$ hormone, despite a significant increase in gonadotropin hormone-related mRNA expression in male zebrafish exposed to BPA alternatives. The first option suggests that the rate of E2 production with the aromatase enzyme is higher than that observed with the T hormone. In contrast, the second option proposes the possibility of decreased expression of several genes involved in steroidogenesis (e.g., star, $17 \beta h s d$, and cyp17).

The commonalities and differences in toxicity of BPA analogs have been explained based on their chemical structure [65,76]. Bisphenols have a phenolic group in common. 
The hydrophobic group of the propane moiety and the 4-hydroxyl group on the A-phenyl ring are suggested regulatory factors that can cause differences in the BPA analog toxicity $[76,77]$. If the estrogenicity and anti-androgenicity of BPA are due to the phenolic ring, the endocrine disruption would be possessed even if BPA analogs are used as substitutes.

\subsection{Effects of BPA and Its Alternatives on HPT Axis}

For elucidating endocrine disruption, bisphenol analogs have primarily been studied focusing on reproductive toxicity, whereas developmental toxicity due to thyroid endocrine disruption has been studied less relatively. Ten studies showed that BPA, BPAF, BPF, BPS, and BPZ influenced hatchability, time-to-hatch, spontaneous movement, thyroid hormone production, and mRNA expression related to the HPT axis (Table 4) [78-87]. 
Table 4. Summary of studies about hypothalamic-pituitary-thyroid (HPT) axis in zebrafish exposed to bisphenol A and its alternatives.

\begin{tabular}{|c|c|c|c|c|c|c|c|c|c|c|}
\hline \multirow[b]{2}{*}{ Chemical } & \multirow[b]{2}{*}{$\begin{array}{l}\text { Stage } \\
\text { (Type) }\end{array}$} & \multirow[b]{2}{*}{$\begin{array}{c}\text { Exposure } \\
\text { Concentration }(\mu \mathrm{g} / \mathrm{L})\end{array}$} & \multirow[b]{2}{*}{$\begin{array}{l}\text { Exposure } \\
\text { Duration }\end{array}$} & \multirow{2}{*}{$\begin{array}{l}\text { No Observed } \\
\text { Adverse Effect } \\
\text { Level } \\
(\mu \mathrm{g} / \mathrm{L})\end{array}$} & \multirow{2}{*}{$\begin{array}{c}\text { Lowest } \\
\text { Observed } \\
\text { Adverse Effect } \\
\text { Level } \\
(\mu \mathrm{g} / \mathrm{L})\end{array}$} & \multicolumn{4}{|c|}{ Toxicity Effect } & \multirow[b]{2}{*}{$\operatorname{Re}$} \\
\hline & & & & & & Gene & Hormone & Organ & Organism & \\
\hline \multirow{2}{*}{ BPA } & \multirow{2}{*}{ Embryo } & \multirow{2}{*}{$\begin{array}{c}0,2,22,228 \\
(=0,0.01,0.1,1 \mu \mathrm{M})\end{array}$} & \multirow{2}{*}{$24 \mathrm{~h}$} & - & 2 & $\uparrow t g, p a x 8$ & - & - & - & \multirow{2}{*}{ [79] } \\
\hline & & & & 2 & 22 & $\uparrow t s h, p a x 2 a$ & - & - & - & \\
\hline BPA & Adult & $0,2,20$ & $4 \mathrm{~min}$ & 2 & 20 & - & $\downarrow \mathrm{T} 4$ (\%) & - & $\downarrow \mathrm{F} 1$ survival & {$[80]$} \\
\hline \multirow{4}{*}{ BPA } & \multirow{4}{*}{ Embryo } & \multirow{4}{*}{$0,80,400,2000,10,000$} & \multirow{4}{*}{$120 \mathrm{~h}$} & - & 80 & $\uparrow$ hhex & - & - & - & \multirow{4}{*}{ [83] } \\
\hline & & & & 80 & 400 & $\uparrow t t r$, dio1, ugt1ab & $\uparrow \mathrm{T} 3$ & - & - & \\
\hline & & & & 2000 & 10,000 & - & - & - & $\downarrow$ Hatchability & \\
\hline & & & & $>10,000$ & - & - & - & - & Length & \\
\hline BPA & Embryo & $0,804,2010,4020,6030$ & $96 \mathrm{~h}$ & - & 804 & $\uparrow t \operatorname{sh} \beta$ & - & - & - & {$[78]$} \\
\hline BPA & $\begin{array}{l}\text { Embryo } \\
\text { (GFP) }\end{array}$ & $0,2282(=0,10 \mu \mathrm{M})$ & $168 \mathrm{~h}$ & $>2282$ & - & $\operatorname{tr} \alpha, \operatorname{tr} \beta$ & - & - & - & [85] \\
\hline \multirow{3}{*}{ BPAF } & \multirow{3}{*}{ Embryo } & \multirow{3}{*}{$0,5,50,500$} & \multirow{3}{*}{$168 \mathrm{~h}$} & - & 5 & $\uparrow t t r$ & $\downarrow$ FT3 & - & - & \multirow{3}{*}{ [84] } \\
\hline & & & & \multirow{2}{*}{5} & \multirow{2}{*}{50} & $\uparrow t s h \beta$, slc5a5, tg, dio1, dio 2 & $\downarrow$ TT4, FT4, TT3 & - & - & \\
\hline & & & & & & $\downarrow \operatorname{tr} \alpha, \operatorname{tr} \beta$ & & & & \\
\hline BPAF & Adult & $0,24.7$ & 21 days & - & 24.7 & $\uparrow t r h, t r h r 1, t \operatorname{sh} \beta, \operatorname{dio} 2\left(\sigma^{\top}\right)$ & - & - & - & [82] \\
\hline & & & & 0.2 & 2 & $\uparrow c r h, t g$ & - & - & - & \\
\hline & & & & 2 & 20 & $\uparrow n i s$, dio2, ugt1ab & $\uparrow \mathrm{TSH}$ & - & - & \\
\hline BPF & Embryo & $0,0.2,2,20,200$ & $144 \mathrm{~h}$ & & & $\downarrow t t r$ & & & & [81] \\
\hline & & & & 20 & 200 & - & $\uparrow \mathrm{T} 3, \downarrow \mathrm{T} 4$ & - & - & \\
\hline & & & & - & 80 & - & - & - & $\uparrow$ Time-to-hatch & \\
\hline BPF & Embryo & $0,80,400,2000,10,000$ & $120 \mathrm{~h}$ & 400 & 2000 & $\uparrow$ hhex, ugt1ab & $\uparrow \mathrm{T} 4$ & - & - & [83] \\
\hline & & & & $>10,000$ & - & - & - & - & Length & \\
\hline & & & & - & 1 & 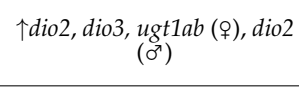 & $\uparrow \mathrm{T} 3(\mathrm{P}), \mathrm{F} 1 \mathrm{~T} 3$ & - & $\begin{array}{c}\downarrow \mathrm{F} 1 \\
\text { spontaneous } \\
\text { movement }\end{array}$ & \\
\hline BPS & Adult & $0,1,10,100$ & 120 days & & & $\downarrow c r h, t \operatorname{sh} \beta\left(0^{\top}\right)$ & $\downarrow \mathrm{T} 4\left(0^{\top}\right.$, $), \mathrm{F} 1 \mathrm{~T} 4$ & - & - & {$[86]$} \\
\hline & & & & 1 & 10 & $\uparrow c r h, \operatorname{dio} 1\left(\sigma^{\top}\right.$, o $)$ & - & - & - & \\
\hline & & & & 10 & 100 & $\uparrow t \operatorname{sh} \beta($ ( $), \operatorname{dio} 3\left(\sigma^{\top}\right)$ & - & - & - & \\
\hline BPS & Fmbryo & $\begin{array}{lllll}0 & 1 & 3 & 10 & 30\end{array}$ & $168 \mathrm{~b}$ & 1 & 3 & $\downarrow t t r$ & - & - & - & [87] \\
\hline BPS & Emoryo & $0,1,3,10,30$ & $168 \mathrm{~h}$ & 3 & 10 & $\uparrow c r h, t g$, dio1, ugt1ab & $\downarrow \mathrm{T} 4, \uparrow \mathrm{TSH}$ & - & - & {$[8 /]$} \\
\hline
\end{tabular}


Table 4. Cont.

\begin{tabular}{|c|c|c|c|c|c|c|c|c|c|c|}
\hline \multirow[b]{2}{*}{ Chemical } & \multirow[b]{2}{*}{$\begin{array}{l}\text { Stage } \\
\text { (Type) }\end{array}$} & \multirow[b]{2}{*}{$\begin{array}{c}\text { Exposure } \\
\text { Concentration }(\mu \mathrm{g} / \mathrm{L})\end{array}$} & \multirow[b]{2}{*}{$\begin{array}{l}\text { Exposure } \\
\text { Duration }\end{array}$} & \multirow{2}{*}{$\begin{array}{l}\text { No Observed } \\
\text { Adverse Effect } \\
\text { Level } \\
(\mu \mathrm{g} / \mathrm{L})\end{array}$} & \multirow{2}{*}{$\begin{array}{c}\text { Lowest } \\
\text { Observed } \\
\text { Adverse Effect } \\
\text { Level } \\
(\mu \mathrm{g} / \mathrm{L}) \\
\end{array}$} & \multicolumn{4}{|c|}{ Toxicity Effect } & \multirow[b]{2}{*}{$\operatorname{Re}$} \\
\hline & & & & & & Gene & Hormone & Organ & Organism & \\
\hline \multirow{4}{*}{ BPS } & \multirow{4}{*}{ Embryo } & \multirow{4}{*}{$0,400,2000,10,000,50,000$} & \multirow{4}{*}{$120 \mathrm{~h}$} & - & 400 & - & - & - & $\uparrow$ Time-to-hatch & \multirow{4}{*}{ [83] } \\
\hline & & & & 400 & 2000 & $\begin{array}{c}\uparrow c r h, \text { tsh } \beta, \text { tshr, hhex, tpo, ttr, } \\
\text { ugt1ab }\end{array}$ & - & - & - & \\
\hline & & & & 10,000 & 50,000 & - & $\uparrow \mathrm{T} 3$ & - & - & \\
\hline & & & & $>50,000$ & - & - & - & - & Length & \\
\hline \multirow{2}{*}{ BPZ } & \multirow{2}{*}{ Embryo } & \multirow{2}{*}{$0,40,180,680,2900$} & \multirow{2}{*}{$120 \mathrm{~h}$} & 180 & 680 & $\uparrow \operatorname{tsh} \beta$ & - & - & - & \multirow{2}{*}{ [83] } \\
\hline & & & & $>2900$ & - & - & - & - & Length & \\
\hline
\end{tabular}




\subsubsection{Effects on Development}

Experimental evidence showed that hatchability, time-to-hatch, eyeball size normalized to the body length, and spontaneous movement were affected by exposure to BPA [80,83], BPF [83], BPS [83,86], and BPZ [83]. Spontaneous movement, any distinguishable movement inside the chorion of embryos, was significantly decreased in fish exposed to environmentally relevant concentration $(1 \mu \mathrm{g} / \mathrm{L})$ of BPS [86]. Especially, significant delay of the time-to-hatch were observed after the exposure to BPA, BPF, BPS, and BPZ [83]. Longer hatching duration of embryos can make them more susceptible to predators and mortality via environmental factors [88]. The effective concentration of BPA was up to 5 25-fold greater than those of BPF or BPS [83]. These results suggest that the endocrine disruption potential of BPF and BPS is no less than that of BPA.

\subsubsection{Effects on Thyroid Hormones and Genes Related to HPT Axis}

Similar to the HPG axis, the thyrotropin-releasing hormone (TRH) with the corticotrophic-releasing hormone (CRH) of the HPT axis secrete thyroid-stimulating hormone (TSH) from the hypothalamus [89]. TSH secreted by the pituitary then regulates the synthesis of thyroid hormone (TH), that is thyroxine (T4) and triiodothyronine (T3). Measurement of thyroid hormones is commonly used in zebrafish, as it is the most integrated endpoint in assessing thyroid endocrine disruption [90]. Zebrafish exposed to BPA [80,83], BPAF [84], BPF [81,83], and BPS [83,86,87] were affected by either activating or suppressing hormone production. Interestingly, significant decreases in $\mathrm{T} 4$ concentrations along with increases in TSH concentration were commonly observed in fish exposed to BPF and BPS [81,87]. Moreover, changes in thyroid hormones were more sensitive to the toxicity of BPS than BPA (Figure 5). These results suggest that the thyroid endocrine disruption of BPA alternatives is no less than that of BPA and may significantly affect on thyroid hormone homeostasis. Decreases in T4 could reduce the TH availability in target tissues and subsequently influence metabolic pathways [78,84].

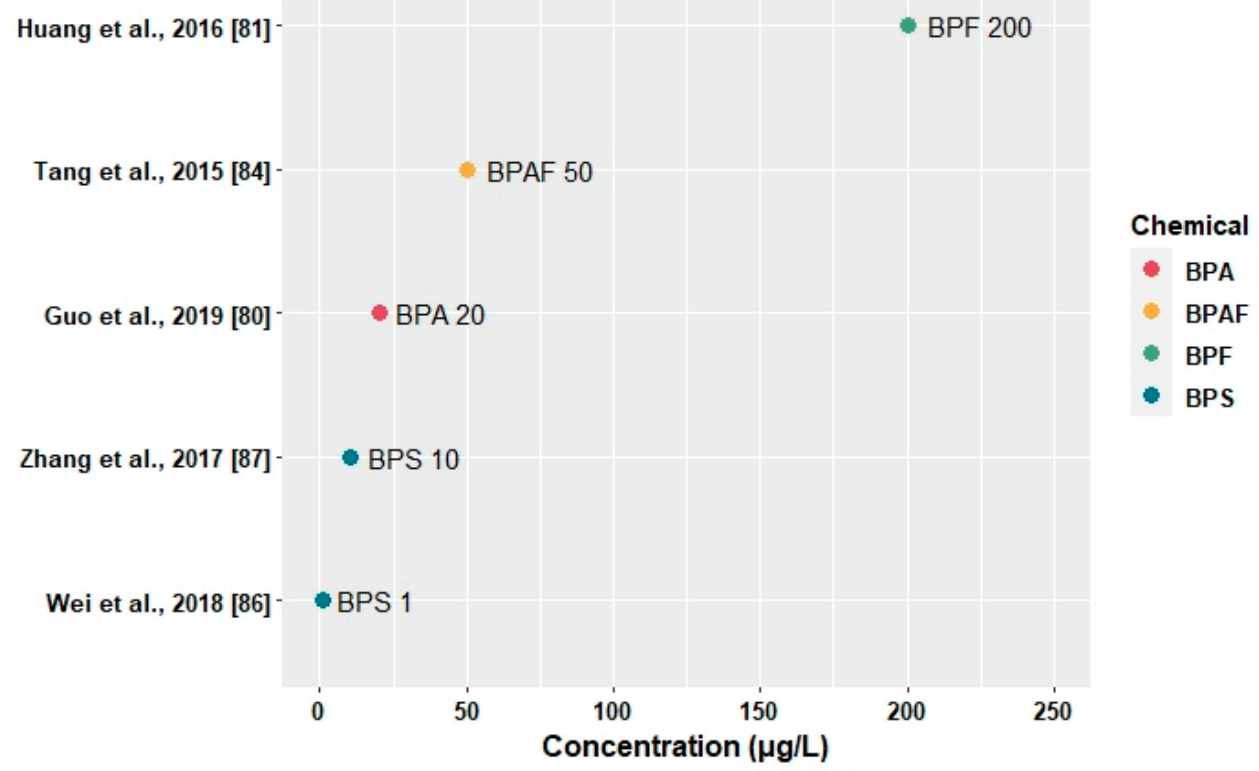

Figure 5. Cont. 
(b) LOAEL of TSH regulation or tsh $\beta$ mRNA expression in zebrafish

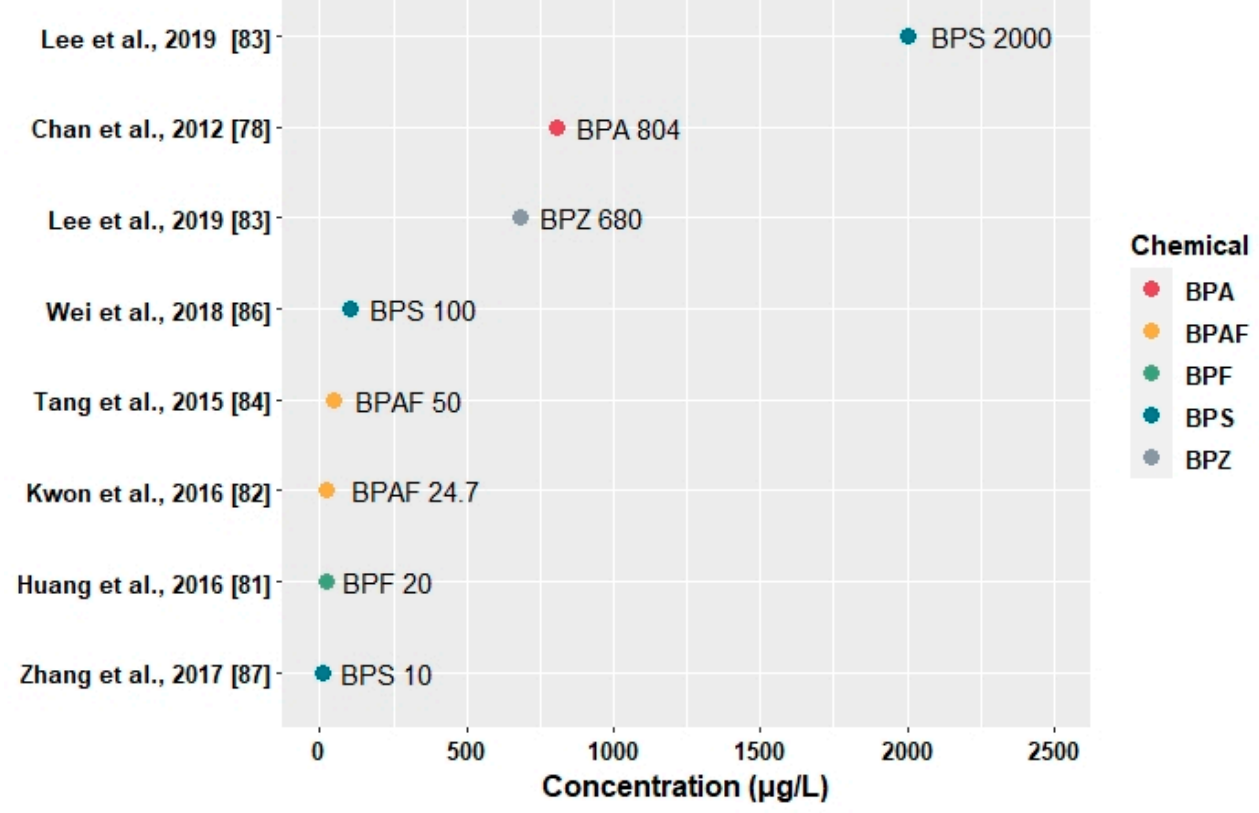

Figure 5. Lowest observed adverse effect level (LOAEL) of thyroxine (T4) hormone (a), thyroid stimulating hormone (TSH) or $t s h \beta$ mRNA expression (b) in zebrafish exposed to BPA, BPAF, BPF, BPS, and BPZ.

TSH has been measured to assess the potential mechanism of thyroid dysfunction. TSH levels increased significantly after BPA analog exposure [81,87], which might be involved in the negative feedback mechanism. Significant up-regulation of $\operatorname{tsh} \beta$ genes is also well supported by changes in TSH levels. TRH and CRH stimulate TSH secretion, and transcription of trh and crh genes were significantly upregulated after exposure to BPAF, BPF, and BPS [81-83,86,87]. The results of these studies suggest that the increase in TSH concentrations and upregulation of trh and crh caused by bisphenol exposure may be attributed to the promotion of thyroid hormone synthesis and its subsequent release to compensate for the decreased T4 levels in zebrafish.

The transcription of various genes in the HPT axis has been measured in zebrafish exposed to BPA, BPAF, BPF, BPS, and BPZ. Iodothyronine deiodinases (Dio) are the key regulators of T4 and T3 bioavailability [91], and three types of dio enzymes (Dio1, Dio2, and Dio3) and genes (dio1, dio2, and dio3) have been found in zebrafish [92]. Dio2 enzyme plays a vital role in catalyzing the conversion of T4 into biologically active T3 [91]. Significant increases in dio2 mRNA expression in zebrafish exposed to BPAF [82,84], BPF [81], and BPS [86] have been reported, and an increase in T3 and a decrease in T4 were observed together in some studies $[81,86]$. These results suggest that BPA analogs potentially affect dio2 gene transcription and thyroid hormone production.

Overall, the results demonstrated that BPA analogs significantly changed thyroid hormone concentrations and modified the mRNA expression of key genes involved in the HPT axis, suggesting thyroid endocrine disruption in zebrafish. BPA analogs and thyroid hormones' chemical structures have similarities; therefore, these chemicals exhibit the property of binding to thyroid receptors and competing with the thyroid hormone [93]. Since endocrine systems interact with each other, further studies are needed to assess BPA alternatives' primary target among the endocrine axes.

\section{Conclusions}

The present study summarized the endocrine-disrupting potential of BPA and its alternatives in zebrafish and identified knowledge gaps. The summary highlights of this study are as follows: 
- $\quad$ BPA alternatives have a similar or more significant toxic potential than that of BPA.

- Several BPA alternatives may cause reproductive dysfunction by interfering in the regulatory mechanisms of the HPG axis or inducing vitellogenin in males.

- Males were more sensitive to the adverse effects on sex hormone levels, as well as gene transcriptions, than females.

- Environmentally relevant concentration of BPA alternatives has the potential to inhibit the normal development of embryo/larvae by disrupting thyroid hormone endocrine system.

The modifications of phenolic rings and bridging carbon or the longer length of the alkyl substitutes seem to influence endocrine-disrupting activity. However, the apparent relationship between their structure and endocrine-disrupting activity was not clarified. Further toxicological information on BPA alternatives is required to understand the environmental health implications of these alternatives and to develop proper management strategies.

Author Contributions: Conceptualization: J.L., K.W.M., K.J.; Formal analysis: J.L., K.J.; Writingoriginal draft: J.L.; Writing-review \& editing: K.W.M., K.J. All authors have read and agreed to the published version of the manuscript.

Funding: This research was funded by the National Research Foundation of Korea (NRF; Project no. 2019R1A2C1002712).

Conflicts of Interest: The authors declare that there is no conflict of interest associated with this work.

\section{References}

1. Kabir, E.R.; Rahman, M.S.; Rahman, I. A review on endocrine disruptors and their possible impacts on human health. Environ. Toxicol. Pharmacol. 2015, 40, 241-258. [CrossRef]

2. Löhr, H.; Hammerschmidt, M. Zebrafish in Endocrine Systems: Recent Advances and Implications for Human Disease. Annu. Rev. Physiol. 2011, 73, 183-211. [CrossRef]

3. USEPA. Available online: https://www.epa.gov/endocrine-disruption/what-endocrine-disruption (accessed on 14 December 2020).

4. OECD. Available online: http:/ /www.oecd.org/chemicalsafety/testing/OECD\%20Work\%20on\%20Endocrine\%20Disrupting\% 20Chemicals.pdf (accessed on 14 December 2020).

5. Lim, M.; Park, J.Y.; Ji, K.; Lee, K. Development and application of a chemical ranking and scoring system for the management of endocrine disrupting chemicals. J. Environ. Health Sci. 2018, 44, 76-89.

6. Gao, X.; Kang, S.; Xiong, R.; Chen, M. Environment-Friendly Removal Methods for Endocrine Disrupting Chemicals. Sustainability 2020, 12, 7615. [CrossRef]

7. Casals-Casas, C.; Desvergne, B. Endocrine Disruptors: From Endocrine to Metabolic Disruption. Annu. Rev. Physiol. 2011, 73, 135-162. [CrossRef] [PubMed]

8. Mathieu-Denoncourt, J.; Wallace, S.J.; de Solla, S.R.; Langlois, V.S. Plasticizer endocrine disruption: Highlighting developmental and reproductive effects in mammals and non-mammalian aquatic species. Gen. Comp. Endocrinol. 2015, 219, 74-88. [CrossRef] [PubMed]

9. Mnif, W.; Hassine, A.I.H.; Bouaziz, A.; Bartegi, A.; Thomas, O.; Roig, B. Effect of Endocrine Disruptor Pesticides: A Review. Int. J. Environ. Res. Public Health 2011, 8, 2265-2303. [CrossRef] [PubMed]

10. Iavicoli, I.; Fontana, L.; Bergamaschi, A. The Effects of Metals as Endocrine Disruptors. J. Toxicol. Environ. Health Part B 2009, 12, 206-223. [CrossRef]

11. Bonefeld-Jørgensen, E.C.; Ghisari, M.; Wielsøe, M.; Bjerregaard-Olesen, C.; Kjeldsen, L.S.; Long, M. Biomonitoring and hormonedisrupting effect biomarkers of persistent organic pollutants in vitro and ex vivo. Basic Clin. Pharmacol. Toxicol. 2014, 115, 118-128. [CrossRef]

12. Rubin, B.S. Bisphenol A: An endocrine disruptor with widespread exposure and multiple effects. J. Steroid Biochem. Mol. Biol. 2011, 127, 27-34. [CrossRef]

13. Huang, Y.; Wong, C.; Zheng, J.; Bouwman, H.; Barra, R.; Wahlstrom, B.; Neretin, L.; Wong, M. Bisphenol A (BPA) in chi-na: A review of sources, environmental levels, and potential human health impacts. Environ. Int. 2012, 42, 91-99. [CrossRef] [PubMed]

14. Kang, J.-H.; Kondo, F.; Katayama, Y. Human exposure to bisphenol A. Toxicology 2006, 226, 79-89. [CrossRef] [PubMed]

15. Cao, X.-L.; Corriveau, J.; Popovic, S. Sources of Low Concentrations of Bisphenol A in Canned Beverage Products. J. Food Prot. 2010, 73, 1548-1551. [CrossRef] 
16. Geens, T.; Goeyens, L.; Kannan, K.; Neels, H.; Covaci, A. Levels of bisphenol-A in thermal paper receipts from Belgium and estimation of human exposure. Sci. Total Environ. 2012, 30-33. [CrossRef]

17. Hengstler, J.G.; Foth, H.; Gebel, T.; Kramer, P.-J.; Lilienblum, W.; Schweinfurth, H.; Völkel, W.; Wollin, K.-M.; Gundert-Remy, U. Critical evaluation of key evidence on the human health hazards of exposure to bisphenol A. Crit. Rev. Toxicol. 2011, 41, $263-291$. [CrossRef]

18. Corrales, J.; Kristofco, L.; Steele, W.; Yates, B.; Breed, C.; Williams, E.; Brooks, B. Global assessment of bisphenol A in the environment: Review and analysis of its occurrence and bioaccumulation. Dose Response 2015, 13, 1-29. [CrossRef]

19. Van den Berg, L.N.; Chahoud, I.; Heindel, J.J.; Padmanabhan, V.; Paumgartten, F.J.R.; Schoenfelder, G. Urinary, circulating, and tissue biomonitoring studies indicate widespread exposure to bisphenol A. Environ. Health Perspect. 2010, 118, 1055-1070. [CrossRef] [PubMed]

20. Zhang, T.; Sun, H.; Kannan, K. Blood and Urinary Bisphenol A Concentrations in Children, Adults, and Pregnant Women from China: Partitioning between Blood and Urine and Maternal and Fetal Cord Blood. Environ. Sci. Technol. 2013, 47, 4686-4694. [CrossRef] [PubMed]

21. Zhang, Z.; Alomirah, H.; Cho, H.-S.; Li, Y.-F.; Liao, C.; Minh, T.B.; Mohd, M.A.; Nakata, H.; Ren, N.; Kannan, K. Urinary Bisphenol A Concentrations and Their Implications for Human Exposure in Several Asian Countries. Environ. Sci. Technol. 2011, 45, 7044-7050. [CrossRef]

22. Wang, W.; Abualnaja, K.; Asimakopoulos, A.; Covaci, A.; Gevao, B.; Johnson-Restrepo, B.; Kumosani, T.; Malarvannan, G.; Minh, T.; Moon, H.; et al. A comparative assessment of human exposure to tetrabromobisphenol A and eight bi-sphenols including bisphenol A via indoor dust ingestion in twelve countries. Environ. Int. 2015, 83, 183-191. [CrossRef]

23. Yamazaki, E.; Yamashita, N.; Taniyasu, S.; Lam, J.; Lam, P.; Moon, H.; Jeong, Y.; Kannan, P.; Achyuthan, H.; Munuswamy, N.; et al. Bisphenol Aa and other bisphenol analogues including BPS and BPF in surface water samples from Japan, China, Korea and India. Ecotoxicol. Environ. Saf. 2015, 122, 565-572. [CrossRef] [PubMed]

24. Moermond, C.T.; Kase, R.; Korkaric, M.; Ågerstrand, M. CRED: Criteria for reporting and evaluating ecotoxicity data. Environ. Toxicol. Chem. 2016, 35, 1297-1309. [CrossRef]

25. Vandenberg, L.N.; Ågerstrand, M.; Beronius, A.; Beausoleil, C.; Bergman, Å.; Bero, L.A.; Bornehag, C.-G.; Boyer, C.S.; Cooper, G.S.; Cotgreave, I.; et al. A proposed framework for the systematic review and integrated assessment (SYRINA) of endocrine disrupting chemicals. Environ. Health 2016, 15, 74. [CrossRef]

26. Klimisch, H.-J.; Andreae, M.; Tillmann, U. A Systematic Approach for Evaluating the Quality of Experimental Toxicological and Ecotoxicological Data. Regul. Toxicol. Pharmacol. 1997, 25, 1-5. [CrossRef]

27. Xia, L.; Zheng, L.; Zhou, J.L. Transcriptional and morphological effects of tamoxifen on the early development of zebrafish (Danio rerio). J. Appl. Toxicol. 2016, 36, 853-862. [CrossRef]

28. Molina, A.; Abril, N.; Morales-Prieto, N.; Monterde, J.; Lora, A.; Ayala, N.; Moyano, R. Evaluation of toxicological end-points in female zebrafish after bisphenol A exposure. Food Chem. Toxicol. 2018, 112, 19-25. [CrossRef]

29. Segner, H. Zebrafish (Danio rerio) as a model organism for investigating endocrine disruption. Comp. Biochem. Physiol. Part C Toxicol. Pharmacol. 2009, 149, 187-195. [CrossRef] [PubMed]

30. Allner, B.; Hennies, M.; Lerche, C.F.; Schmidt, T.; Schneider, K.; Willner, M.; Stahlschmidt-Allner, P. Kinetic determination of vitellogenin induction in the epidermis of cyprinid and perciform fishes: Evaluation of sensitive enzyme-linked immunosorbent assays. Environ. Toxicol. Chem. 2016, 35, 2916-2930. [CrossRef]

31. Chen, H.; Zhao, L.; Yu, Q. Determination and reduced life expectancy model and molecular docking analyses of estro-genic potentials of 17beta-estradiol, bisphenol A and nonylphenol on expression of vitellogenin gene (vtg1) in zebrafish. Chemosphere 2019, 221, 727-734. [CrossRef]

32. Chen, L.; Hu, C.; Guo, Y.; Shi, Q.; Zhou, B. Tio2 nanoparticles and BPA are combined to impair the development of off-spring zebrafish after parental coexposure. Chemosphere 2019, 217, 732-741. [CrossRef] [PubMed]

33. Chen, M.; Zhang, J.; Pang, S.; Wang, C.; Wang, L.; Sun, Y.; Song, M.; Liang, Y. Evaluating estrogenic and anti-estrogenic effect of endocrine disrupting chemicals (EDCs) by zebrafish (Danio rerio) embryo-based vitellogenin 1 (vtg1) mRNA ex-pression. Comp. Biochem. Physiol. C Toxicol. Pharmacol. 2018, 204, 45-50. [CrossRef]

34. Chen, W.; Lau, S.; Fan, Y.; Wu, R.; Ge, W. Juvenile exposure to bisphenol A promotes ovarian differentiation but sup-presses its growth - potential involvement of pituitary follicle-stimulating hormone. Aquat. Toxicol. 2017, 193, 111-121. [CrossRef] [PubMed]

35. Chow, W.S.; Chan, W.K.-L.; Chan, K.M. Toxicity assessment and vitellogenin expression in zebrafish (Danio rerio) embryos and larvae acutely exposed to bisphenol A, endosulfan, heptachlor, methoxychlor and tetrabromobisphenol A. J. Appl. Toxicol. 2013, 33, 670-678. [CrossRef] [PubMed]

36. Fang, Q.; Shi, Q.; Guo, Y.; Hua, J.; Wang, X.; Zhou, B. Enhanced bioconcentration of bisphenol A in the presence of nano-tio2 can lead to adverse reproductive outcomes in zebrafish. Environ. Sci. Technol. 2016, 50, 1005-1013. [CrossRef]

37. Ji, K.; Seo, J.; Kho, Y.; Choi, K. Co-exposure to ketoconazole alters effects of bisphenol A in Danio rerio and H295R cells. Chemosphere 2019, 237, 124414. [CrossRef] [PubMed]

38. Kausch, U.; Alberti, M.; Haindl, S.; Budczies, J.; Hock, B. Biomarkers for exposure to estrogenic compounds: Gene ex-pression analysis in zebrafish (Danio rerio). Environ. Toxicol. 2008, 23, 15-24. [CrossRef]

39. Keiter, S.; Baumann, L.; Färber, H.; Holbech, H.; Skutlarek, D.; Engwall, M.; Braunbeck, T. Long-term effects of a binary mixture of perfluorooctane sulfonate (PFOS) and bisphenol A (BPA) in zebrafish (Danio rerio). Aquat. Toxicol. 2012, 116-129. [CrossRef] 
40. $\quad$ Laing, L.; Viana, J.; Dempster, E.L.; Trznadel, M.; Trunkfield, L.A.; Webster, T.M.U.; Van Aerle, R.; Paull, G.C.; Wilson, R.J.; Mill, J.; et al. Bisphenol A causes reproductive toxicity, decreases dnmt1 transcription, and reduces global DNA methylation in breeding zebrafish (Danio rerio). Epigenetics 2016, 11, 526-538. [CrossRef]

41. Liu, Y.; Yao, Y.; Li, H.; Qiao, F.; Wu, J.; Du, Z.-Y.; Zhang, M. Influence of Endogenous and Exogenous Estrogenic Endocrine on Intestinal Microbiota in Zebrafish. PLOS ONE 2016, 11, e0163895. [CrossRef]

42. Mu, X.; Huang, Y.; Li, X.; Lei, Y.; Teng, M.; Li, X.; Wang, C.; Li, Y. Developmental Effects and Estrogenicity of Bisphenol A Alternatives in a Zebrafish Embryo Model. Environ. Sci. Technol. 2018, 52, 3222-3231. [CrossRef]

43. Muncke, J.; Junghans, M.; Eggen, R.I.L. Testing estrogenicity of known and novel (xeno-)estrogens in the MolDarT using developing zebrafish (Danio rerio). Environ. Toxicol. 2007, 22, 185-193. [CrossRef] [PubMed]

44. Olsvik, P.; Whatmore, P.; Penglase, S.; Skjaerven, K.; Angles d'Auriac, M.; Ellingsen, S. Associations between behavioral effects of bisphenol A and DNA methylation in zebrafish embryos. Front. Genet. 2019, 10, 184. [CrossRef]

45. Pinto, C.; Hao, R.; Grimaldi, M.; Thrikawala, S.; Boulahtouf, A.; Aït-Aïssa, S.; Brion, F.; Gustafsson, J.-Å.; Balaguer, P.; Bondesson, M. Differential activity of BPA, BPAF and BPC on zebrafish estrogen receptors in vitro and in vivo. Toxicol. Appl. Pharmacol. 2019, 380, 114709. [CrossRef] [PubMed]

46. Qiu, W.; Fang, M.; Liu, J.; Fu, C.; Zheng, C.; Chen, B.; Wang, K. In vivo actions of bisphenol F on the reproductive neu-roendocrine system after long-term exposure in zebrafish. Sci. Total Environ. 2019, 665, 995-1002. [CrossRef] [PubMed]

47. Qiu, W.; Shao, H.; Lei, P.; Zheng, C.; Qiu, C.; Haiyang, S.; Zheng, Y. Immunotoxicity of bisphenol S and F are similar to that of bisphenol A during zebrafish early development. Chemosphere 2018, 194, 1-8. [CrossRef]

48. Qiu, W.; Zhao, Y.; Yang, M.; Farajzadeh, M.; Pan, C.; Wayne, N. Actions of bisphenol A and bisphenol S on the reproduc-tive neuroendocrine system during early development in zebrafish. Endocrinology 2016, 157, 636-647. [CrossRef] [PubMed]

49. Santangeli, S.; Consales, C.; Pacchierotti, F.; Habibi, H.; Carnevali, O. Transgenerational effects of BPA on female re-production. Sci. Total Environ. 2019, 685, 1294-1305. [CrossRef] [PubMed]

50. Santangeli, S.; Maradonna, F.; Gioacchini, G.; Cobellis, G.; Piccinetti, C.; Dalla Valle, L.; Carnevali, O. BPA-induced de-regulation of epigenetic patterns: Effects on female zebrafish reproduction. Sci. Rep. 2016, 6, 21982. [CrossRef]

51. Song, M.; Liang, D.; Liang, Y.; Chen, M.; Wang, F.; Wang, H.; Jiang, G. Assessing developmental toxicity and estrogenic activity of halogenated bisphenol A on zebrafish (Danio rerio). Chemosphere 2014, 112, 275-281. [CrossRef]

52. Sun, S.; Zhang, Y.; Lu, D.; Wang, W.; Limbu, S.; Chen, L.; Zhang, M.; Du, Z. Concentration-dependent effects of 17beta-estradiol and bisphenol A on lipid deposition, inflammation and antioxidant response in male zebrafish (Danio rerio). Chemosphere 2019, 237, 124422. [CrossRef]

53. Van den Belt, K.; Verheyen, R.; Witters, H. Comparison of vitellogenin responses in zebrafish and rainbow trout fol-lowing exposure to environmental estrogens. Ecotoxicol. Environ. Saf. 2003, 56, 271-281. [CrossRef]

54. Villeneuve, D.L.; Garcia-Reyero, N.; Escalon, B.L.; Jensen, K.M.; Cavallin, J.E.; Makynen, E.A.; Durhan, E.J.; Kahl, M.D.; Thomas, L.M.; Perkins, E.J.; et al. Ecotoxicogenomics to Support Ecological Risk Assessment: A Case Study with Bisphenol A in Fish. Environ. Sci. Technol. 2011, 46, 51-59. [CrossRef] [PubMed]

55. Wang, J.; Shi, X.; Du, Y.; Zhou, B. Effects of xenoestrogens on the expression of vitellogenin (vtg) and cytochrome p450 aromatase (cyp19a and b) genes in zebrafish (Danio rerio) larvae. J. Environ. Sci. Health A Tox. Hazard. Subst. Environ. Eng. 2011, 46, 960-967. [CrossRef]

56. Zhao, F.; Wei, P.; Wang, J.; Yu, M.; Zhang, X.; Tian, H.; Wang, W.; Ru, S. Estrogenic effects associated with bisphenol A exposure in male zebrafish (Danio rerio) is associated with changes of endogenous 17beta-estradiol and gene specific DNA methylation levels. Gen. Comp. Endocrinol. 2017, 252, 27-35. [CrossRef] [PubMed]

57. Shi, J.; Jiao, Z.; Zheng, S.; Li, M.; Zhang, J.; Feng, Y.; Yin, J.; Shao, B. Long-term effects of bisphenol AF (BPAF) on hor-monal balance and genes of hypothalamus-pituitary-gonad axis and liver of zebrafish (Danio rerio), and the impact on offspring. Chemosphere 2015, 128, 252-257. [CrossRef]

58. Yang, X.; Liu, Y.; Li, J.; Chen, M.; Peng, D.; Liang, Y.; Song, M.; Zhang, J.; Jiang, G. Exposure to Bisphenol AF disrupts sex hormone levels and vitellogenin expression in zebrafish. Environ. Toxicol. 2016, 31, 285-294. [CrossRef]

59. Le Fol, V.; Ait-Aissa, S.; Sonavane, M.; Porcher, J.; Balaguer, P.; Cravedi, J.; Zalko, D.; Brion, F. In vitro and in vivo es-trogenic activity of BPA, BPF and BPS in zebrafish-specific assays. Ecotoxicol. Environ. Saf. 2017, 142, 150-156. [CrossRef]

60. Yang, Q.; Yang, X.; Liu, J.; Chen, Y.; Shen, S. Effects of exposure to BPF on development and sexual differentiation during early life stages of zebrafish (Danio rerio). Comp. Biochem. Physiol. Part C Toxicol. Pharmacol. 2018, 210, 44-56. [CrossRef]

61. Yang, Q.; Yang, X.; Liu, J.; Ren, W.; Chen, Y.; Shen, S. Effects of BPF on steroid hormone homeostasis and gene expres-sion in the hypothalamic-pituitary-gonadal axis of zebrafish. Environ. Sci. Pollut. Res. Int. 2017, 24, 21311-21322. [CrossRef]

62. Ji, K.; Hong, S.; Kho, Y.; Choi, K. Effects of Bisphenol S Exposure on Endocrine Functions and Reproduction of Zebrafish. Environ. Sci. Technol. 2013, 47, 8793-8800. [CrossRef] [PubMed]

63. Naderi, M.; Salahinejad, A.; Attaran, A.; Chivers, D.; Niyogi, S. Chronic exposure to environmentally relevant concen-trations of bisphenol S differentially affects cognitive behaviors in adult female zebrafish. Environ. Pollut. 2020, 261, 114060. [CrossRef]

64. Naderi, M.; Wong, M.Y.; Gholami, F. Developmental exposure of zebrafish (Danio rerio) to bisphenol-S impairs subsequent reproduction potential and hormonal balance in adults. Aquat. Toxicol. 2014, 148, 195-203. [CrossRef]

65. Lee, J.; Park, N.-Y.; Kho, Y.; Ji, K. Effects of 4-Hydroxyphenyl 4-Isoprooxyphenylsulfone (BPSIP) Exposure on Reproduction and Endocrine System of Zebrafish. Environ. Sci. Technol. 2018, 52, 1506-1513. [CrossRef] 
66. Ma, Y.; Han, J.; Guo, Y.; Lam, P.K.; Wu, R.S.; Giesy, J.P.; Zhang, X.; Zhou, B. Disruption of endocrine function in in vitro H295R cell-based and in in vivo assay in zebrafish by 2,4-dichlorophenol. Aquat. Toxicol. 2012, 106-107, 173-181. [CrossRef]

67. Liu, C.; Deng, J.; Yu, L.; Ramesh, M.; Zhou, B. Endocrine disruption and reproductive impairment in zebrafish by expo-sure to 8:2 fluorotelomer alcohol. Aquat. Toxicol. 2010, 96, 70-76. [CrossRef] [PubMed]

68. Hutchinson, T.; Ankley, G.; Segner, H.; Tyler, C. Screening and testing for endocrine disruption in fish-biomarkers as "signposts," not "traffic lights," in risk assessment. Environ. Health Perspect. 2006, 114, 106-114. [CrossRef] [PubMed]

69. Belt, K.V.D.; Verheyen, R.; Witters, H. Reproductive Effects of Ethynylestradiol and 4t-Octylphenol on the Zebrafish (Danio rerio). Arch. Environ. Contam. Toxicol. 2001, 41, 458-467. [CrossRef] [PubMed]

70. Yang, F.-X.; Xu, Y.; Hui, Y. Reproductive effects of prenatal exposure to nonylphenol on zebrafish (Danio rerio). Comp. Biochem. Physiol. Part C Toxicol. Pharmacol. 2006, 142, 77-84. [CrossRef] [PubMed]

71. Peteffi, G.P.; Fleck, J.D.; Kael, I.M.; Rosa, D.C.; Antunes, M.V.; Linden, R. Ecotoxicological risk assessment due to the presence of bisphenol A and caffeine in surface waters in the Sinos River Basin - Rio Grande do Sul-Brazil. Braz. J. Biol. 2019, 79, 712. [CrossRef]

72. Kallivretaki, E.; Eggen, R.; Neuhauss, S.; Alberti, M.; Kausch, U.; Segner, H. Aromatase in zebrafish: A potential target for endocrine disrupting chemicals. Mar. Environ. Res. 2006, 62, S187-S190. [CrossRef]

73. Trant, J.M.; Gavasso, S.; Ackers, J.; Chung, B.-C.; Place, A.R. Developmental expression of cytochrome P450 aromatase genes (CYP19a and CYP19b) in zebrafish fry (Danio rerio). J. Exp. Zool. 2001, 290, 475-483. [CrossRef] [PubMed]

74. Schally, A.; Arimura, A.; Kastin, A.; Matsuo, H.; Baba, Y.; Redding, T.; Nair, R.; Debeljuk, L.; White, W. Gonadotro-pin-releasing hormone: One polypeptide regulates secretion of luteinizing and follicle-stimulating hormones. Science 1971, 173, 1036-1038. [CrossRef] [PubMed]

75. Tello, J.A.; Wu, S.; Rivier, J.E.; Sherwood, N.M. Four functional GnRH receptors in zebrafish: Analysis of structure, signaling, synteny and phylogeny. Integr. Comp. Biol. 2008, 48, 570-587. [CrossRef]

76. Ji, K.; Choi, K. Endocrine disruption potentials of bisphenol A alternatives - are bisphenol A alternatives safe from en-docrine disruption? Korean J. Environ. Health Sci. 2013, 39, 1-18. [CrossRef]

77. Kitamura, S.; Suzuki, T.; Sanoh, S.; Kohta, R.; Jinno, N.; Sugihara, K.; Yoshihara, S.; Fujimoto, N.; Watanabe, H.; Ohta, S. Comparative Study of the Endocrine-Disrupting Activity of Bisphenol A and 19 Related Compounds. Toxicol. Sci. 2005, 84, 249-259. [CrossRef]

78. Chan, W.; Chan, K. Disruption of the hypothalamic-pituitary-thyroid axis in zebrafish embryo-larvae following wa-terborne exposure to BDE-47, TBBPA and BPA. Aquat. Toxicol. 2012, 108, 106-111. [CrossRef] [PubMed]

79. Gentilcore, D.; Porreca, I.; Rizzo, F.; Ganbaatar, E.; Carchia, E.; Mallardo, M.; De Felice, M.; Ambrosino, C. Bisphenol A interferes with thyroid specific gene expression. Toxicology 2013, 304, 21-31. [CrossRef] [PubMed]

80. Guo, Y.; Chen, L.; Wu, J.; Hua, J.; Yang, L.; Wang, Q.; Zhang, W.; Lee, J.; Zhou, B. Parental co-exposure to bisphenol A and nano-Tio2 causes thyroid endocrine disruption and developmental neurotoxicity in zebrafish offspring. Sci. Total Environ. 2019, 650, 557-565. [CrossRef] [PubMed]

81. Huang, G.-M.; Tian, X.-F.; Fang, X.-D.; Ji, F.-J. Waterborne exposure to bisphenol F causes thyroid endocrine disruption in zebrafish larvae. Chemosphere 2016, 147, 188-194. [CrossRef] [PubMed]

82. Kwon, B.; Kho, Y.; Kim, P.-G.; Ji, K. Thyroid endocrine disruption in male zebrafish following exposure to binary mixture of bisphenol AF and sulfamethoxazole. Environ. Toxicol. Pharmacol. 2016, 48, 168-174. [CrossRef]

83. Lee, S.; Kim, C.; Shin, H.; Kho, Y.L.; Choi, K. Comparison of thyroid hormone disruption potentials by bisphenols A, S, F, and Z in embryo-larval zebrafish. Chemosphere 2019, 221, 115-123. [CrossRef]

84. Tang, T.; Yang, Y.; Chen, Y.; Tang, W.; Wang, F.; Diao, X. Thyroid Disruption in Zebrafish Larvae by Short-Term Exposure to Bisphenol AF. Int. J. Environ. Res. Public Health 2015, 12, 13069-13084. [CrossRef] [PubMed]

85. Terrien, X.; Fini, J.; Demeneix, B.; Schramm, K.; Prunet, P. Generation of fluorescent zebrafish to study endocrine disrup-tion and potential crosstalk between thyroid hormone and corticosteroids. Aquat. Toxicol. 2011, 105, 13-20. [CrossRef] [PubMed]

86. Wei, P.; Zhao, F.; Zhang, X.; Liu, W.; Jiang, G.; Wang, H.; Ru, S. Transgenerational thyroid endocrine disruption induced by bisphenol $S$ affects the early development of zebrafish offspring. Environ. Pollut. 2018, 243, 800-808. [CrossRef]

87. Zhang, D.-H.; Zhou, E.-X.; Yang, Z.-L. Waterborne exposure to BPS causes thyroid endocrine disruption in zebrafish larvae. PLoS ONE 2017, 12, e0176927. [CrossRef]

88. Ong, K.J.; Zhao, X.; Thistle, M.E.; MacCormack, T.J.; Clark, R.J.; Ma, G.; Martinez-Rubi, Y.; Simard, B.; Loo, J.S.C.; Veinot, J.G.; et al. Mechanistic insights into the effect of nanoparticles on zebrafish hatch. Nanotoxicology 2014, 8, 295-304. [CrossRef] [PubMed]

89. Orozco, A.; Valverde-R, C. Thyroid Hormone Deiodination in Fish. Thyroid 2005, 15, 799-813. [CrossRef]

90. Zaccaroni, A.; Gamberoni, M.; Mandrioli, L.; Sirri, R.; Mordenti, O.; Scaravelli, D.; Sarli, G.; Parmeggiani, A. Thyroid hormones as a potential early biomarker of exposure to 4-nonylphenol in adult male shubunkins (Carassius auratus). Sci. Total Environ. 2009, 407, 3301-3306. [CrossRef]

91. Houbrechts, A.; Delarue, J.; Gabriels, I.; Sourbron, J.; Darras, V. Permanent deiodinase type 2 deficiency strongly per-turbs zebrafish development, growth, and fertility. Endocrinology 2016, 157, 3668-3681. [CrossRef] 
92. Castañeda Cortés, D.; Langlois, V.; Fernandino, J. Crossover of the hypothalamic pituitary-adrenal/interrenal-thyroid, andgonadal axes in testicular development. Front. Endocrinol. 2014, 5, 139. [CrossRef]

93. Iyigündoğdu, I.; Üstündağ, A.; Duydu, Y. Toxicological Evaluation of Bisphenol A and Its Analogues. Turk. J. Pharm. Sci. 2020, 17, 457-462. [CrossRef] [PubMed] 